University of Massachusetts Amherst

ScholarWorks@UMass Amherst

October 2021

\title{
Early Development of ADHD and ODD Symptoms from the Toddler to Preschool Years
}

Hallie Brown

University of Massachusetts Amherst

Follow this and additional works at: https://scholarworks.umass.edu/dissertations_2

Part of the Child Psychology Commons

\section{Recommended Citation}

Brown, Hallie, "Early Development of ADHD and ODD Symptoms from the Toddler to Preschool Years" (2021). Doctoral Dissertations. 2277.

https://doi.org/10.7275/22977392 https://scholarworks.umass.edu/dissertations_2/2277

This Open Access Dissertation is brought to you for free and open access by the Dissertations and Theses at ScholarWorks@UMass Amherst. It has been accepted for inclusion in Doctoral Dissertations by an authorized administrator of ScholarWorks@UMass Amherst. For more information, please contact scholarworks@library.umass.edu. 
Early Development of ADHD and ODD Symptoms from the Toddler to Preschool Years

A Dissertation Presented

by

HALLIE R. BROWN

Submitted to the Graduate School of the University of Massachusetts Amherst in partial fulfillment of the requirements for the degree of

Doctor of Philosophy

September 2021

Psychological and Brain Sciences 
C by Hallie R. Brown 2021

All Rights Reserved 
Early Development of ADHD and ODD Symptoms from the Toddler to Preschool Years

A Dissertation Presented

by

HALLIE R. BROWN

Approved as to style and content by:

Elizabeth A. Harvey, Chair

Kirby D. Deater-Deckard, Member

Sara A. Whitcomb, Member

Holly B. Laws, Member

Caren Rotello, Department Head

Psychological and Brain Sciences 


\begin{abstract}
EARLY DEVELOPMENT OF ADHD AND ODD SYMPTOMS FROM THE TODDLER TO PRESCHOOL YEARS
\end{abstract}

SEPTEMBER 2021

\author{
HALLIE BROWN, B.A., HAMILTON COLLEGE \\ M.S., UNIVERSITY OF MASSACHUSETTS AMHERST \\ PH.D., UNIVERSITY OF MASSACHUSETTS AMHERST
}

Directed by: Professor Elizabeth A. Harvey

ADHD and ODD are common and impairing externalizing disorders in childhood that are often comorbid. Understanding the development of these symptoms when they first emerge is crucial for better identifying children who are at-risk for later impairment. Parents $(\mathrm{N}=273)$ were recruited via Amazon's Mechanical Turk to complete surveys about their 2-year-old, followed by surveys every six months for two years. Children's general pattern of ADHD and ODD symptom growth were examined with latent class analyses. Temperament traits were subsequently added as covariates. Dual group-based trajectories and cross-lagged analyses examined the co-development of these disorders. Results showed 4 distinct growth trajectories for each symptom domain. Most children follow low symptom trajectories of ODD and each domain of ADHD. A small portion of children show high symptoms across time, and others show moderate or increasing symptoms. Only the hyperactive/impulsive domain of ADHD showed a declining symptoms group akin to the "terrible twos." Temperament traits of low effortful control, high negative affect, and high surgency increased odds of membership in high symptom groups. Children with high ODD symptoms across time had a high likelihood of 
membership in high ADHD symptom groups; the converse was true but probabilities were lower. Finally, hyperactive/impulsive symptoms predicted ODD symptoms across time, more than vice versa, while ODD predicted inattention symptoms. The study extends the small body of literature assessing the early development of ADHD and ODD and uses DSM-based symptoms to enhance clinical utility. Findings suggest that some children with early symptoms of ADHD or ODD are at risk for continued symptomatology and developing comorbid symptoms. 


\section{TABLE OF CONTENTS}

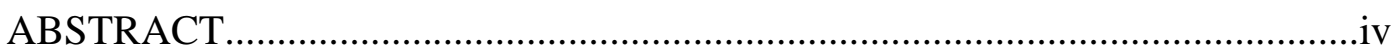

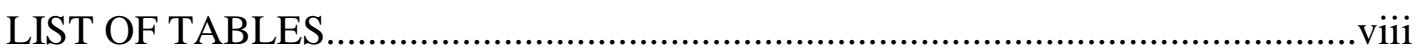

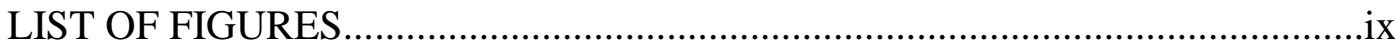

\section{CHAPTER}

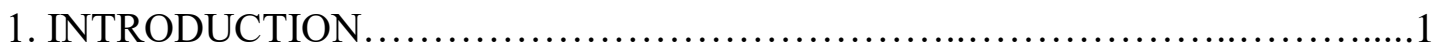

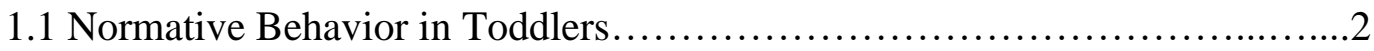

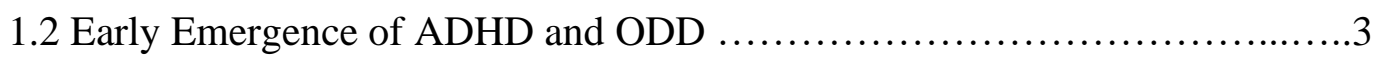

1.2.1 Cross sectional research..............................................

1.2.2 Longitudinal Studies..........................................

1.3 Temperament Risk for ADHD and ODD …..............................

1.4 Co-Development of ADHD and ODD .................................... 10

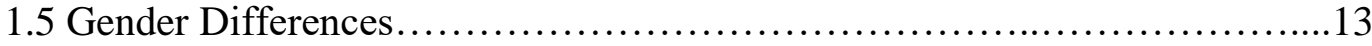

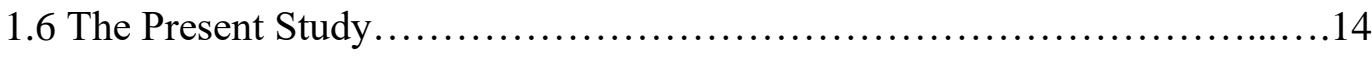

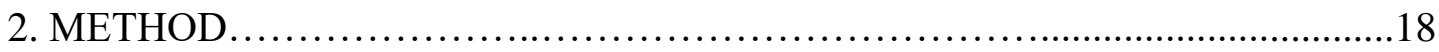

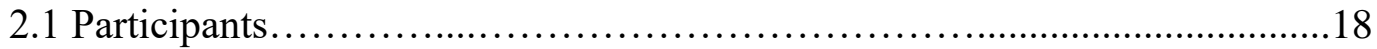

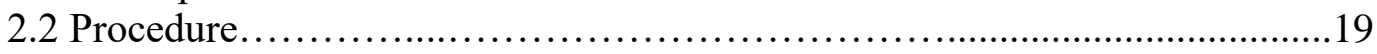

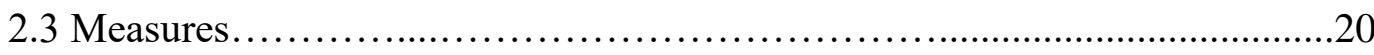

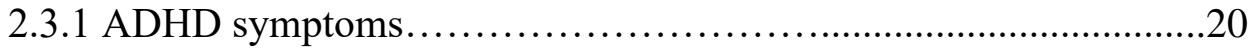

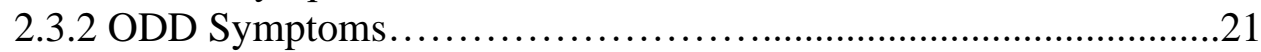

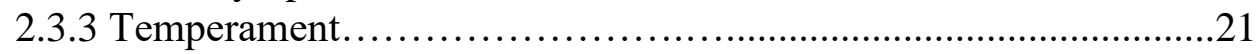

2.3.3 Demographics and Family History ..................................................22

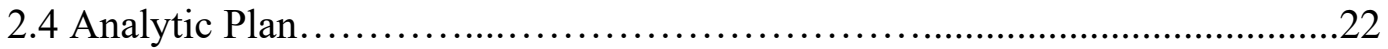

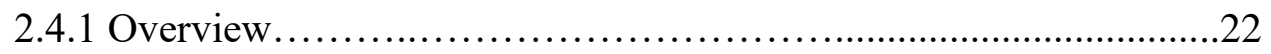

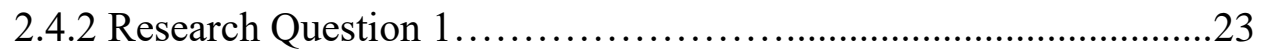

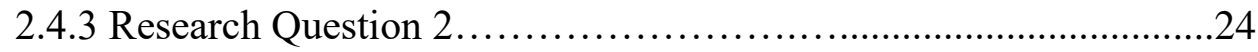

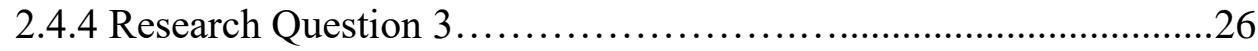

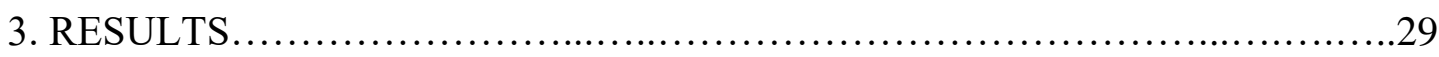

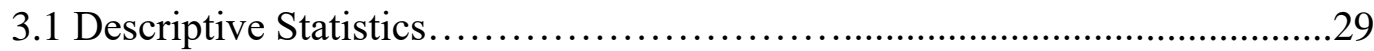


3.2 Research Question 1............................................................... 30

3.2.1 Hyperactivity/Impulsivity .......................................................30

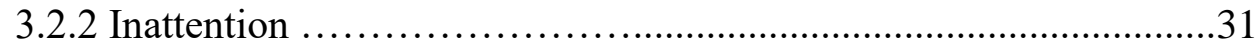

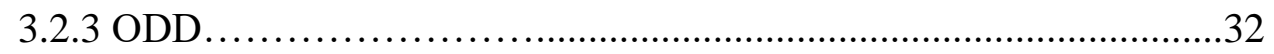

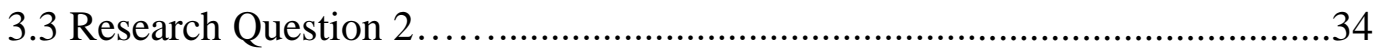

3.3.1 Hyperactivity/Impulsivity .......................................................34

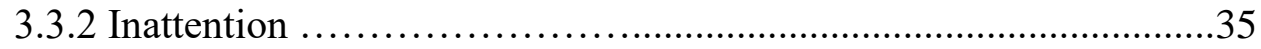

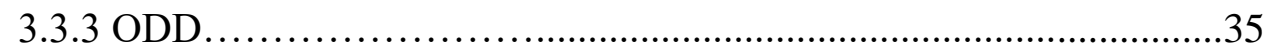

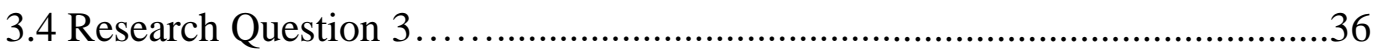

3.4.1 Dual Trajectories ....................................................................36

3.4.2 Hyperactivity and ODD Cross-Lagged Models ..........................38

3.4.3 Inattention and ODD Cross-Lagged Models ......................................39

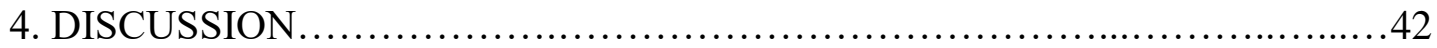

4.1 What are Common Trajectories of DSM Symptoms of ADHD and ODD

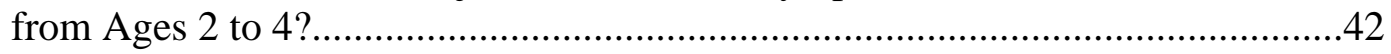

4.1.1 Hyperactivity/Impulsivity .......................................................42

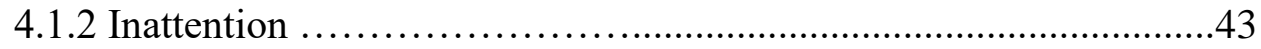

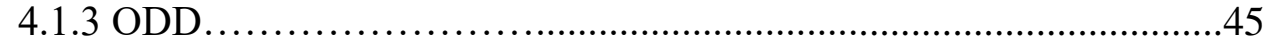

4.1.4 Gender Differences in Growth................................................46

4.2 Does Toddler Temperament Distinguish Among Trajectory Groups?............48

4.3 How do Symptom Trajectories of ADHD and ODD Co-vary?........................51

4.3.1 Dual Trajectories ......................................................................51

4.3.2 Cross-Lagged Effects..............................................................53

4.3.3 Theory Underlying Co-development.................................................55

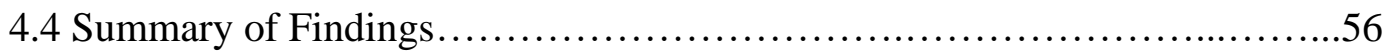

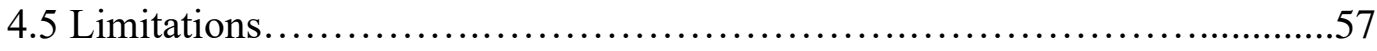

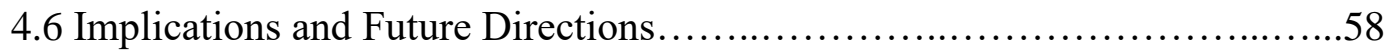

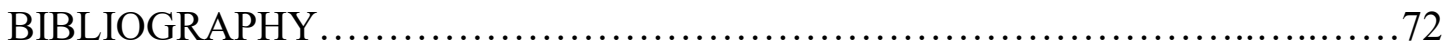




\section{LIST OF TABLES}

Table

Page

1. ADHD and ODD Average Symptom Ratings and Symptom Counts..............65

2. Fit Statistics for Trajectories of ADHD and ODD..................................66

3. Summary of Final Trajectory Models......................................67

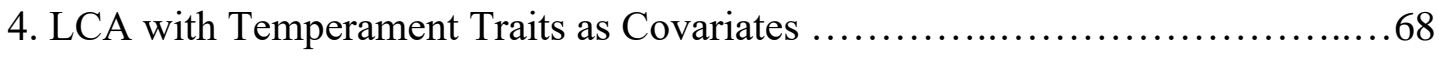

5. Dual Trajectory Results for ADHD and ODD Trajectory Classes.....................70

6. Dual Trajectory Results for Hyperactivity/impulsivity and Inattention Classes.....71 


\section{LIST OF FIGURES}

Figure

Page

1. Hyperactivity/impulsivity Trajectories from Time 1 to Time $5 \ldots \ldots \ldots \ldots \ldots \ldots \ldots$

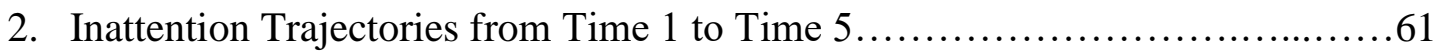

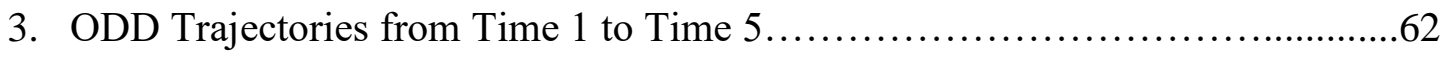

4. Hyperactive Effects Model between Hyperactivity and ODD, with Fixed paths

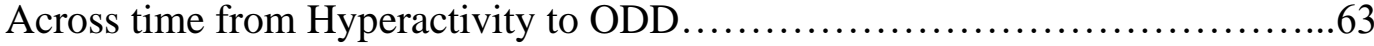

5. Cross-lagged Model between Inattention and ODD, with Fixed Paths across Time from ODD to Inattention............................................64 


\section{CHAPTER 1}

\section{INTRODUCTION}

Attention-deficit/hyperactivity disorder (ADHD) and oppositional defiant disorder (ODD) are two common externalizing disorders in children that can cause substantial functional impairment across multiple domains (e.g., de la Osa et al., 2019; Gupte-Singh et al., 2017; Halvorsen et al., 2019; Johnston \& Mash, 2001). ADHD is characterized by developmentally deviant symptoms in one or both of two domains: inattention (e.g., distractibility, disorganization) and/or hyperactivity/impulsivity (e.g., overactivity, interrupting). Etiology of ADHD is thought to be largely biological; genetic risk factors contribute to neural (e.g., pre-frontal cortex) and neurotransmitter (e.g., dopaminergic) network abnormalities, and reduced volume in certain brain regions (e.g., Faraone et al., 2015; Sharma \& Couture, 2015). However, environmental factors such as hostile parenting styles, parent psychopathology, exposure to toxins, and deprivation are also implicated in symptom development (e,g., Bornovalova et al., 2010; Faraone et al., 2015). ODD involves frequent hostile and defiant behaviors that span three domains: irritability, argumentativeness, and/or vindictiveness. The disorder is also thought to have both genetic and environmental (e.g., parent psychopathology, family drug use) causes (e.g., Bornovalova et al., 2010), though there is suggestion that ODD is more influenced by environmental factors than ADHD (Azeredo et al., 2018). ADHD and ODD are highly comorbid; about $40 \%$ of children with ADHD also have ODD (e.g., Elia et al., 2008), perhaps due in part to their overlapping risk factors.

Signs of ADHD and ODD often emerge early in life and both disorders can be diagnosed as young as preschool (American Psychiatric Association, APA, 2013). 
Research has shown that symptoms are fairly stable starting in preschool, with around 7590\% continuing to meet criteria several years later (e.g., Lahey et al., 2004; Riddle et al., 2013). However, compared to research on school-age children, the early emergence of symptoms of these disorders is less well studied. Better understanding the early development of these disorders is essential for early intervention and prevention. One key area of investigation is differentiating the early emergence of symptoms from typical toddler developmental phases (i.e., "terrible twos"). It is important not to overpathologize a normal developmental phase, yet identifying children who may show stable high levels of these behaviors could lead to better early interventions to optimize prognosis, similar to efforts with other neurodevelopmental disorders such as autism spectrum disorder. Early identification of these disorders requires a better understanding of the common patterns of symptom development that children follow in early childhood, including the way these two highly comorbid disorders co-develop. Further, identifying early factors such as temperament traits that can predict these patterns will help elucidate which children may at risk for more chronic symptomatology versus which children are in a transient developmental phase.

\subsection{Normative Behavior in Toddlers}

Identifying early signs of psychopathology in toddlerhood is challenging because toddlers are in a developmentally normative phase characterized by behavior resembling externalizing symptoms, including high activity and oppositional behavior. Toddlers are commonly defiant, argumentative, and temperamental (e.g., Campbell, 1990; Kochanska et al., 2001; Wakschlag et al., 2012) and observational studies have found high rates of parent-child arguing at this age (e.g., Dix et al., 2007). These behaviors are thought to 
occur because toddlers are learning to exert their autonomy (e.g., Dix et al., 2007;

Erikson, 1963) and have limited abilities of self-regulation and behavioral control (e.g., Kochanska et al., 2001; Rothbart et al., 2011). Distinguishing between emerging psychopathology and normative development is thus challenging due to the nature of typical behavior at this age. However, although these behaviors may be common features of the toddler years, not all toddlers age out of this phase. It is possible that for some toddlers, these behaviors are not "terrible twos" and instead represent the onset of more serious externalizing symptoms. A growing body of literature suggests that some toddlers show significant behavior problems beyond typical toddlerhood, and importantly, a small body of longitudinal studies suggest that not all toddlers "grow out" of this developmental phase and in fact are at risk for chronic symptomatology. Understanding patterns of these symptoms beginning in toddlerhood may help differentiate typical toddlerhood from early signs of behavior disorders.

\subsection{Early Emergence of ADHD and ODD}

\subsubsection{Cross sectional research}

Although behavior problems are common in toddlers, there appears to be a subset of toddlers who show severe levels of ADHD- and ODD-like symptoms distinct from normative developmental behavior. Several studies have found that a portion of children as young as 2 years old show clinically elevated levels of ADHD and ODD symptoms, suggesting that there are some toddlers who display externalizing psychopathology beyond what might be developmentally appropriate. For instance, Lavigne and colleagues (1996) found that $12.6 \%$ of 2-year-olds met criteria for ODD, with $7.1 \%$ showing severe cases, while $2 \%$ of community children age 2-5 met criteria for ADHD (though rates for 
2-year-olds alone were not reported). Among clinic-referred children (i.e., children whose parents have behavioral concerns), rates are higher. Keenen \& Wakschlag (2000) found that almost $80 \%$ of parent-referred toddlers and preschoolers met criteria for externalizing disorders, with 59.5\% meeting criteria for ADHD and 59.5\% meeting criteria for ODD, though estimates were not presented separately for toddlers. Fox and colleagues (2007) found that among toddlers referred to a low-income mental health clinic, $48.1 \%$ showed ODD and a small portion (1.9\%) presented with ADHD. These studies indicate that some toddlers show clinically significant levels of these symptoms, and further, that some children's behavior is severe enough to motivate parents to seek professional help. Additionally, there is some evidence that toddlers displaying severe levels of these behaviors are distinguishable from typical toddlers. Seminal work by Campbell and colleagues in the 1980s found that clinic-referred toddlers with behavior problems could be distinguished from control children with $88 \%$ accuracy, using parentreport and laboratory measures of behavior (Campbell et al., 1982). Taken together, these studies suggest some children may display high levels of ADHD and ODD symptoms in toddlerhood, and that parents and clinicians distinguish these young children from peers. However, these cross-sectional studies do not establish if these early difficulties are transient, which requires longitudinal research.

\subsubsection{Longitudinal Studies}

A smaller body of literature suggests that a portion of children who show high symptoms of ADHD or ODD in toddlerhood continue to show impairing symptoms over time. Other children show a reduction in symptomatology, suggesting they "grow out" of a developmental phase. In a series of seminal studies, Campbell and colleagues followed 
two cohorts of externalizing 2- to 3-year-old children (Campbell et al., 1982). At age 6, half of the children with earlier behavior problems showed persistent symptoms, with one third meeting criteria for ADHD and $15 \%$ showing clinically significant aggression (Campbell et al., 1986). At age 9, 52\% (15 out of 29 children) showed persistent problems, as reported by parents and teachers (Campbell \& Ewing, 1990), with almost $20 \%$ meeting criteria for ADHD and just over $25 \%$ meeting criteria for ODD or Conduct Disorder (CD; Pierce et al., 1999). In adolescence, a majority of the children continued to show clinically relevant behavior problems, with over $50 \%$ meeting criteria for ADHD and over $40 \%$ for ODD/CD (Pierce et al., 1999). Children who showed persistent problems during childhood were even more likely to have disorders in adolescence than children with less persistent problems, suggesting that early onset and chronic symptoms confer the greatest risk for later externalizing disorders. Other studies have similarly found that toddlers with behavior problems show stability in symptoms; for instance, $70 \%$ of children with disruptive behavior disorders at ages 2-5 still showed these disorders about 2 years later (Lavigne et al., 1998), though rates specifically for 2-yearolds were not provided.

A handful of trajectory-based studies have elucidated common growth patterns of these symptoms, and have provided additional evidence that toddlers with particularly high levels of ADHD and ODD symptoms continue to show stable levels of these symptoms across early childhood. A small portion of toddlers show chronic ODD-like behavior (e.g., aggression and conduct problems), with rates ranging from $4 \%$ to $25 \%$ of children falling in the chronic symptoms group (e.g., Côté et al., 2006; Huijbregtset al., 2007; Petitclerc et al., 2009; Shaw et al., 2003; Shaw et al., 2005; Tremblay et al., 2004). 
Similar studies of ADHD have shown that a small portion of toddlers with severe symptoms continue to show high symptoms across early childhood; rates vary across studies from 2\% to 20\%, though most are under $8 \%$ (Galéra et al., 2011; Huijbregts et al., 2007; Leblanc et al., 2008; Palfrey et al., 1985; Romano et al., 2006; Salla et al., 2016; Shaw et al., 2005). Thus, although rates vary, a substantial minority of toddlers show elevated symptoms of ADHD and ODD that continue across early childhood. These trajectory studies also identify other common growth patterns, including a portion of children who show symptom remittance, others who show more moderate symptomatology but may show impairment later, and others who show few symptoms across time. More thorough evaluation of these trajectories can help clarify the typical growth patterns of these symptoms, including improving early identification of a clinically high-risk group.

Expanding these trajectory studies is important due to a number of limitations in the current literature. Given the lack of diagnostic measures validated for use at this age, these longitudinal studies rely on a small number of non-DSM based items to measure symptoms, such as a handful of items from a child behavior checklist. The small number of symptoms used limits the clinical utility of these findings, particularly since ADHD and ODD are heterogeneous disorders with individuals showing different combinations of symptoms. Relatedly, ADHD studies mostly examine the hyperactive/impulsive domain but not the inattentive domain, with some exceptions (e.g., Galán et al., 2019; Galéra et al., 2011; Salla et al., 2016). Research suggests ODD is multidimensional, consisting of multiple distinct but related domains (e.g., Burke et al., 2010; Burke et al., 2014; Stringaris \& Goodman, 2009); however, many studies simply example a handful of 
symptoms such as aggression rather than all DSM-based symptoms. Findings from the first wave of data collection for the present study suggest that DSM-based symptoms of both domains of ADHD can be applied to the toddler years (Brown \& Harvey, 2018), but most studies have not examined all DSM symptoms of these two externalizing disorders and how they change over time, thus limiting our understanding of the severity and chronicity of symptoms. Despite these limitations, past studies suggest that there are children who are not simply in a toddlerhood defiant and overactive stage, but that they continue to show high levels of externalizing symptoms.

\subsection{Temperament Risk for ADHD and ODD}

Temperament refers to an individual's reactivity and self-regulation, which is thought to have biological underpinnings yet is also malleable by environmental factors (Rothbart et al., 2000). Reactivity includes excitability and arousal whereas selfregulation refers to modulating one's reactivity (Rothbart et al., 2000). Temperament traits are thought to be related to psychopathology, though the nature of this relation has been debated. One theory posits that psychopathology represents extreme versions of temperament traits (spectrum model; Tacket, 2006). However, some findings refute this

theory; for instance, Nigg et al. (2002) found that temperament traits only explain half of the variance in ADHD symptoms. Others have proposed a vulnerability model (Tacket, 2006) which suggests that certain temperament traits predispose individuals to develop psychopathology.

A large body of literature has linked temperament traits to the development of ADHD and ODD symptoms. Traits of poor effortful control, high surgency/extraversion, and high reactivity, particularly negative affect, are related to later ADHD and ODD 
symptoms (e.g., Kochanska \& Knaack, 2003; Murray \& Kochanska, 2002; Rubin et al., 2003; Stifter et al., 2008; Stringaris et al., 2010; Wichstrøm et al., 2018). However, although multiple temperament domains have been linked to each disorder, theory also suggests some specificity of traits to later symptom domains. Martel (2009) proposes several models of temperament risk for ADHD and ODD. These theoretical models generally suggest that low effortful control confers risk specifically for the inattentive domain of ADHD due to the relevance of cognitive control and top-down processing to these symptoms. In contrast, poor reactive control, including more extreme positive and negative emotions, is proposed to confer risk for the hyperactive/impulsive domain of ADHD which is characterized by more emotion dysregulation and bottom-up processing. Poor reactive control is also theorized to confer risk for ODD, though Martel (2009) suggests this may be via hyperactive/impulsive symptoms of ADHD. There is limited empirical research examining this theoretical model, but some studies have found such specificity. For instance, in children and adolescents, traits representing top-down processing or effortful control (e.g., response inhibition, conscientiousness) relate more strongly to inattentive symptoms while reactive or bottom-up processing traits (e.g., negative emotion, agreeableness) relate more strongly to hyperactive/impulsive symptoms (Martel et al., 2009). Further, although multiple domains predict ODD, negative affect is a more consistent predictor of symptoms than other temperament domains (Wichstrøm et al., 2018). More research is needed to build on this small body of research to clarify how different temperament traits predispose children towards ADHD and ODD.

In addition to examining how temperament predicts the level of symptoms of 
psychopathology, some studies have examined how temperament predicts growth of symptoms. That is, temperament may confer risk for the development of psychopathology and whether symptoms remain chronic versus represent a transient stage. The small number of studies examining how temperament traits predict growth (i.e., trajectories) of externalizing symptoms reveal mixed findings. For instance, difficult temperament has been shown to predict steeper decline in externalizing symptoms, likely related to starting with a large number of symptoms (e.g., Miner \& Clarke-Stewart, 2008). In contrast, declining effortful control in adolescents predicted increasing ADHD and ODD symptoms over time (Atherton et al., 2019), whereas in younger children, infant temperament did not relate to rate of change of externalizing symptoms (Owens \& Shaw, 2003). Other studies suggest difficult temperament is related to worse outcome, but due to moderating effects on the growth of symptoms: toddlers with difficult temperament were more vulnerable to influence by environmental factors (e.g., parenting, parent psychopathology) in terms of their risk and protective factors for later externalizing symptoms, whereas children with easier temperament did not show such vulnerability (e.g., Kochanska \& Kim, 2013; Mesman et al., 2009; Owens \& Shaw, 2003). More research is needed to clarify the nature of the relationship between temperament and growth of externalizing disorders. Importantly, studies are needed to examine how toddler temperament traits confer risk of ADHD and ODD symptom trajectories using the DSM-based criteria (i.e., not a broadband externalizing measure). Such analyses are important for informing our understanding of the specificity of temperament risk on psychopathology and differentiating between children likely to show chronic symptoms from those in a developmental phase. 


\subsection{Co-Development of ADHD and ODD}

High comorbidity between ADHD and ODD has been well-documented, but the reasons for their overlap are not well-understood. It has been theorized that overlap may be due to correlated risk factors, with both disorders stemming from a shared etiology (Rhee et al., 2008), such as common genetic factors (e.g., Harvey et al., 2016; Kerekes et al., 2014; Tuvblad et al., 2009). In particular, earlier onset of comorbidity is thought to be related strongly to a shared genetic influence (see Azeredo et al., 2018). However, researchers have also argued that ADHD and ODD may be causally related to each other; studies have found that children with ADHD are at risk for ODD symptoms, with some evidence that this relation is mediated by factors such as family stress or parenting practices (e.g., Harvey et al., 2016; Kaiser, et al., 2010). Longitudinal research that examines how ADHD and ODD change together and predict one another over time during early development is important toward elucidating a causal connection between the development of ADHD symptoms and ODD symptoms. Dual trajectory and crosslagged models are two statistical approaches to analyzing longitudinal data that are wellsuited to examining these connections.

Group-based dual trajectory models allow us to examine whether children on certain trajectories of one type of disorder are likely to follow specific trajectories of other disorders. In contrast to relying on correlations between constructs (e.g., correlation between inattention and ODD), these models take into account the heterogeneity in developmental trajectories, since correlations may not apply similarly to all individuals (Nagin \& Tremblay, 2001). Past studies examining such dual trajectories have found that children following low trajectories of hyperactivity or conduct problems similar to ODD 
(e.g., aggression, tantrums) were likely to follow low trajectories of the other symptom domain; likewise, chronic high trajectories were linked (e.g., Fontaine et al., 2008; Shaw et al., 2005), suggesting growth of these disorders is related. Similarly, Jester and colleagues $(2005,2008)$ found that some children are on chronic trajectories of both aggression and inattention/hyperactivity, which puts them at risk for worse outcomes. They also found that some children were only on the chronic trajectory of one disorder and not the other, which conferred less risk for poor outcomes. These studies further indicate that growth of these disorders may be positively related at least for some children. However, there are some important gaps in the literature. For example, it is important to extend findings to other symptoms of ODD instead of just aggression, and to examine separate domains of ADHD rather than combining symptoms from a broadband measure (e.g., Jester et al., 2008). Additionally, few studies have examined younger children (e.g., Shaw et al., 2005), an important gap because disorders may be first emerging at this time and thus there may be greater benefit of intervention to alter trajectories. A more thorough examination of the ways risk trajectories intersect for these disorders might help elucidate the way these two disorders change together as symptoms develop in early childhood.

Cross-lagged analyses can be used to examine the directional nature of ADHD and ODD across time; that is, they can examine whether symptoms of one disorder predict symptoms of the other at subsequent time points, controlling for earlier symptoms. Several studies have utilized cross-lagged analyses to examine the relation between ADHD and ODD or related symptoms, although mostly in older children after initial symptoms may have emerged. These studies examining the bidirectional 
relationship between ADHD and ODD have generally provided evidence that ADHD is more predictive of ODD than vice versa. For example, across three time points in a twoyear period of elementary schoolers, ADHD was more predictive of ODD than ODD was predictive of ADHD (Burns \& Walsh, 2002). Across school-age through adolescence, Burke et al., (2005) found that ADHD symptoms, specifically hyperactive/impulsive but not inattentive symptoms, predicted subsequent ODD symptoms; the reverse direction was reported only as marginally significant. One study in preschoolers (Harvey et al., 2016) similarly suggests that the specific domain of symptoms might matter to directionality. At different time points, each domain of ADHD (hyperactive/impulsive and inattention) predicted subdomains of ODD: argumentative/defiant and anger/irritability. In the reverse direction, only the argumentative/defiance domain of ODD predicted inattentive symptoms, and only for boys. This finding supports the need to examine gender differences in bidirectional relations. Further, Harvey et al. (2016) was, to my knowledge, the only study examining cross-lagged relations between ADHD and ODD in young children; other studies have focused on older children, and none on toddlers. It is possible that the directional relations differ by age. Studying the bidirectional relation between ADHD and ODD when these symptoms first emerge in toddlerhood will help clarify the causal nature and ultimately suggest directions for intervention to prevent comorbid symptoms.

In sum, understanding the nature of how ADHD and ODD symptoms co-develop and influence each other over time is important because outcomes are notably worse when children have both disorders (e.g., Galán et al., 2019; Shaw et al., 2001). By better understanding how these disorders co-develop, researchers can hopefully develop 
targeted interventions to reduce co-occurring symptoms and impairment. Evaluating symptom growth using all diagnostic symptoms (i.e., Diagnostic and Statistical Manual of Mental Disorders, $5^{\text {th }}$ edition, DSM-5; APA, 2013) will provide a more precise measure of trajectories than using a handful of symptoms from behavior rating scales. Overall, longitudinal studies suggest that growth of these disorders may be related, but this is a small body of literature and limited by the lack of focus on DSM-based symptoms. Extending this literature to toddlers and using symptoms that are part of the diagnostic criteria is imperative for better understanding the relation between these symptoms in early childhood.

\subsection{Gender Differences}

Symptoms of ADHD and ODD have been found to be more prevalent in boys than girls, though findings are somewhat mixed depending on age. As such, when examining these disorders, it is important to consider potential gender differences. Crosssectional studies show that boys have more symptoms of ADHD compared to girls during the school-age years (DuPaul et al., 2016). This difference is less consistent in younger years and may be smaller. In one study, preschool boys and girls showed no statistical difference in the number of ADHD symptoms (McGoey et al., 2007). In contrast, Lavigne et al. (2009) found that more preschool boys met criteria for ADHD inattentive presentation than girls, though other subtypes of ADHD did not show gender differences. In the first wave of the present study, there was a small effect where 2-year-old boys showed more hyperactive/impulsive symptoms than girls, though there were no gender differences in inattentive symptoms (Brown \& Harvey, 2018). Like ADHD, ODD is more prevalent in boys in middle childhood (e.g., ages 5 to 15; see Demmer et al., 2017 
for review). Although toddler boys may show more aggression than girls (e.g., Rubin et al., 2003), overall rates of ODD in preschoolers appear no different across genders (Lavigne et al., 2009), suggesting that gender differences may be smaller in younger children. Overall, there is mixed evidence as to whether rates of ADHD and ODD are higher for younger boys and girls. Trajectory-based studies show gender differences such that boys are more likely to be on a chronic high-risk trajectory of ODD (e.g., Petitcler et al., 2009) and ADHD (e.g., Romano et al., 2006) symptoms compared to girls. Temperament risk factors may also differentially affect genders, though there are mixed findings. For example, the relation between toddler effortful control and preschool externalizing problems was present for boys but not girls in one study (Smith \& Day, 2018), though showed no gender differences in another study (Rubin et al., 2003). Thus, risk for and trajectories of ADHD and ODD symptoms may not be uniform for boys and girls, though more research is needed given the mixed findings.

\subsection{The Present Study}

The present study examines the longitudinal development of symptoms of ADHD and ODD across two years beginning in toddlerhood (age 2). I aim to address an important gap in the literature by examining trajectories of ADHD and ODD using DSMbased symptoms, to enhance clinical utility. Given their high comorbidity, evaluating these two disorders simultaneously will help elucidate how the development of symptoms of one disorder relates to symptoms of the other. Research on the overlap between these disorders is mixed and generally focuses on older children, without using symptoms aligned with diagnostic criteria. Understanding early signs of these disorders is crucial for identifying children who may benefit from early intervention, particularly high-risk 
children. Specific research questions I address are as follows:

\section{What are common trajectories of DSM symptoms of ADHD and ODD from} ages 2 to 4? This study builds on existing longitudinal research to identify distinct developmental pathways of DSM-based symptoms of ADHD and ODD. I estimated group-based trajectories for ADHD (inattentive and hyperactive/impulsive domains) and $\mathrm{ODD}^{1}$ symptoms to identify models that best represent symptom growth over time. I expected that for inattention, hyperactivity/impulsivity, and ODD, a small portion of children, representing children displaying early emergence of ADHD/ODD, would show a high stable level of symptoms starting in the toddler years. A second group of children, representing children growing out of normative toddler behaviors, were predicted to show declining symptom levels. A third group of children, reflecting children who may be at risk for later onset of $\mathrm{ADHD} / \mathrm{ODD}$, were expected to show moderate or increasing levels of symptomatology. Finally, I expected that a portion of children, representing children who are at low risk for psychopathology, would show a low non-clinical level over time. I also tested whether there are gender differences in the proportion of children in each trajectory. Given the higher prevalence of ADHD and ODD symptoms in boys compared to girls, I expected that the proportions in each trajectory would differ by gender, with more boys in chronic high-risk or moderate symptom groups and more girls in improving and low-risk groups.

\footnotetext{
${ }^{1}$ Although ODD can be broken into subdomains, these domains have 2-3 symptoms each and thus were unstable for the complex analyses in this study.
} 


\section{Does toddler temperament distinguish among trajectory groups? I tested}

whether toddlerhood temperament traits predicted membership in growth trajectories. Past research findings suggest that temperament traits of low effortful control and high surgency predict later ADHD and ODD symptoms, with high negative affect possibly more strongly predictive of ODD symptoms than the other temperament domains (Wichstrøm et al., 2018). Although findings predicting growth have been mixed, based on theory (Martel, 2009) I expected the temperament trait of effortful control would predict early and sustained elevations in inattention (i.e., high stable or increasing symptoms groups). In contrast, traits of high surgency and high negative affect, generally representing high reactivity, were expected to predict early and sustained elevations in hyperactivity/impulsivity. High negative affect was also expected to predict early and sustained symptoms ODD.

\section{How do symptom trajectories of ADHD and ODD covary over time?}

Examining how ADHD and ODD symptoms covary across early development is important for better understanding how comorbidity between these disorders develops. In particular, evaluating how these symptoms change together over time and teasing apart the bidirectional influences of these symptoms on one another can inform theoretical models of comorbidity. First, I used group-based dual trajectories to examine how trajectory membership in one domain (e.g., hyperactivity/impulsivity) was associated with trajectory membership in another domain (e.g., ODD). I expected that children on chronic high-risk trajectories for either domain would be more likely to be in the chronic high-risk trajectory of the 
other domain, and vice versa. Second, I examined the bidirectional influences of ADHD and ODD on one another using a series of cross-lagged models of symptoms across two years ( 5 times points), to better understand whether symptoms of one disorder confer risk for the other disorder. Past literature suggests that ADHD symptoms may confer risk for ODD via impact on factors such as parenting and family stress (Harvey et al., 2016; Kaiser et al., 2010). Thus, I expected that ADHD domains would be generally more predictive of ODD than vice versa. It was expected that both domains of ADHD contribute to ODD symptoms. However, the hyperactivity/impulsivity domain of ADHD was expected to be a stronger predictor of ODD symptoms compared to the inattention domain, given findings that this domain particularly disrupts family processes (e.g., parenting) that contribute to ODD symptoms (e.g., Nelson et al., 2019). ODD was expected to be less predictive of ADHD than vice versa. However, it is possible that ODD may predict ADHD differently for boys and girls, similar to past findings that some subdomains of ODD predicted inattentive symptoms for boys, but not girls (Harvey et al., 2016). 


\section{CHAPTER 2}

\section{METHOD}

\subsection{Participants}

Participants/guardians were parents $(N=273)$ of young children $(157$ boys; 116 girls) who were recruited online via Amazon's Mechanical Turk (MTurk) to complete surveys about their 2-year-old children (Time 1) and follow-up surveys every six months for 2 years, for a total of five time points. Participants were drawn from a larger study; parents/guardians in the present study completed Time 1 and at least one additional time point. $^{2}$ At Time 1 , children were 2 years old $(M=29.32$ months, $S D=3.53)$, followed by Time $2(M=35.38$ months, $S D=3.69)$, Time $3(M=41.46$ months, $S D=3.53)$, Time 4 ( $M=47.39$ months, $S D=3.77)$, and Time $5(M=53.37$ months, $S D=3.60)$. Parents/guardians included 218 biological mothers, 51 biological fathers, 2 step or adoptive mothers, and 3 step or adoptive fathers. Most (72.5) toddlers were White, with a small portion (10.6\%) identified as multi-racial, Black (5.1\%), Asian (1.8\%), or American Indian (.7\%). In regard to ethnicity, $11.3 \%$ of toddlers were identified as Latino/Hispanic. Participants resided in 47 states across the United States. Parents were almost all (99\%) high school graduates, with an additional $48.2 \%$ achieving at least a bachelor's degree. We excluded toddlers with chronic genetic or medical conditions or those who had diagnosed or suspected autism or intellectual disability at Time 1. A small portion of parents at Time 1 reported that their child had a diagnosed (1.1\%) or suspected

\footnotetext{
${ }^{2}$ Children whose parents did not complete a follow-up did not differ from children with follow-ups on any temperament or psychopathology variable, although they were on average 1 month younger than those who had at least a second time point; $t(417)=2.90$, $p=.004$.
} 
(2.5\%) developmental or verbal delay. A small portion of parents (4.7\%) suspected their toddler might have ADHD at Time 1.

\subsection{Procedure}

Participants were recruited via MTurk to allow for recruitment of a more geographically diverse and representative sample than a local sample would yield. MTurk is increasingly used in clinical psychology (e.g., Chandler \& Shapiro, 2016) and past studies have shown that data from MTurk is reliable and comparable to in-person data collection (e.g., Buhrmester, Kwang, \& Goslin, 2011; Schleider \& Weisz, 2015). Details of data collection can be found in Brown and Harvey (2018). Briefly, MTurk users anonymously completed a screening survey about their family for a study called "Parents Perceptions of Young Children's Behavior.” Those parents with 2-year-olds were then invited to complete the full survey; this two-step procedure was to minimize users claiming that they had a child in our target age range (age 2), since they did not know what demographics we were recruiting. The full survey was comprised of a consent form and several questionnaires, with attention check questions (e.g., "Please select answer choice 3") and repeated demographics throughout the survey to check for consistency of responses and attention. Parents received $\$ 0.02$ for the screen survey and $\$ 1.50$ for participating, consistent with current MTurk rates.

In order to achieve a large enough sample, 4,835 MTurk workers were screened, of which 708 (14.6\%) reported they were parents of 2-year-old children. Participants who completed the full survey were excluded for the following reasons: different birthdays reported at follow-ups $(N=91)$, inconsistent Time 1 survey responses (e.g., missing attention check questions, inconsistent demographics from repeated survey questions; $N$ 
$=123)$; not answering at least $25 \%$ of either measure used in present analyses $(N=5)$; twins, because they could not be distinguished at follow-up for the larger study $(N=4)$; diagnosis of or suspected autism, intellectual disability, cerebral palsy, or Down syndrome $(N=51)$. We had a final Time 1 sample of 434 parents of 2 -year-olds (see Brown \& Harvey, 2018). Every six months for two years, parents were contacted through MTurk inviting them to complete a follow-up survey (also paid $\$ 1.50$ for participation). For the present study, we only included parents who completed at least 1 additional follow-up, yielding a final sample of 273 parents of toddlers (all completed Time 1, 198 completed Time 2, 162 completed Time 3, 146 completed Time 4, and 138 completed Time 5).

\subsection{Measures}

\subsubsection{ADHD Symptoms}

ADHD symptoms were measured at each time point with the ADHD Rating Scale-IV Preschool Version (McGoey et al., 2007). The rating scale includes all 18 DSMIV-TR symptoms, with slight adaptations of examples for preschoolers; the symptoms are highly similar to the DSM-5. Parents rate the frequency of each symptom over the last 6 months from 0 (never) to 4 (very often). The measure has previously shown good internal consistency with preschoolers (ATT $\alpha=.93, \mathrm{H} / \mathrm{I} \alpha=.92$, and Total $\alpha=.95$ ), and correlations with other behavior rating scales ( $r=.54$ to .96 ; McGoey et al., 2007). In the first wave of data collection for this study, the rating scale was shown to have good psychometric properties in 2-year-olds (particularly for nonverbal items), including factor analysis, item response theory, and convergent/divergent validity with a temperament measure (Brown \& Harvey, 2018). In the present sample, internal consistency was good 
at each time point (Time 1: $\alpha=.90$; Time 2: $\alpha=.91$; Time 3: $\alpha=.91$; Time 4: $\alpha=.93$; Time 5: $\alpha=.94)$. A two-factor model of ADHD with inattention and hyperactive/impulsive factors (each containing 9 symptoms) was used for all ADHD analyses, based on previous findings with this sample (Brown \& Harvey, 2018).

\subsubsection{ODD Symptoms}

The Disruptive Behavior Rating Scale ODD section (Barkley, 1998) was administered at all time points. This rating scale consists of eight symptoms from the DSM-IV-TR diagnostic criteria for ODD (almost identical to the DSM-5 besides the order of symptoms). Parents report the frequency of each symptom over the last 6 months from 0 (never) to 3 (very often). In a study of preschoolers using the entire disruptive behavior rating scale, which includes ADHD symptoms, the ODD rating scale showed good internal consistency ( $\alpha=.86$ to .89 depending on informant and number of factors; Friedman-Weieneth et al., 2009). In the present study, internal consistency was good at each time point (Time 1: $\alpha=.80$; Time 2: $\alpha=.87$; Time 3: $\alpha=.88$; Time 4: $\alpha=.88$; Time 5: $\alpha=.89$ ). There are mixed findings regarding how many subdomains of ODD symptoms exist, with some studies finding one factor (e.g., Cavanagh et al., 2017; Hommerson et al., 2006), bifactor (Burke et al., 2014) or three-factor models to be the best fit (e.g., Burke et al., 2010, Stringaris and Goodman, 2009). Findings in the present sample indicated that Burke and colleagues' (2010) three-factor model was the best fit for ODD symptoms, however, models for primary research questions were too unstable with domains of two to three symptoms, so analyses were conducted collapsing all ODD symptoms into one domain.

\subsubsection{Temperament}


At Time 1, parents completed The Early Childhood Behavior Questionnaire Short Form (Putnam et al., 2010) a 107-item temperament questionnaire for ages 18 to 36 months. Parents rated the frequency of behavior on a 7-point Likert scale ranging from 1 (never) to 7 (always). The scale comprises 18 temperament traits, clustered into three factors that will be used for the present study: Negative Affect, Surgency/Extraversion, and Effortful Control. Past studies have found moderate to high internal consistency of subscales (ranging .65 to $.83 ; M=.74$ ) and stability over 6 -month $(.58), 12$-month $(.53)$, and 18-month (.46) periods (Putnam et al., 2010). The present sample showed moderate to high internal consistency for the three temperament domains (negative affect $\alpha=.69$, effortful control $\alpha=.74$. and surgency $\alpha=.53$ ).

\subsubsection{Demographics and Family History}

Parents completed demographic questions about their family, including reporting diagnosed or suspected psychiatric and medical conditions and family history of ADHD. They were also asked to identify concerns about their child's behavior and whether their child had been evaluated previously.

\subsection{Analytic Plan}

\subsubsection{Overview}

Mplus Version 8 (Muthén \& Muthén, 2017) was used for all analyses. There were up to five time points of ADHD and ODD symptom ratings for each child; everyone in the final sample had at least two data points of ADHD and ODD symptoms. Temperament ratings were collected at Time 1 for all children. Full information maximum likelihood was used to address missing data. Descriptive statistics were conducted prior to primary analyses to ensure that model assumptions of variable 
distributions were met. To test research question 1 evaluating common trajectories of ADHD and ODD from ages 2 to 4, latent class analyses (LCA) were used. To test research question 2, evaluating how temperament predicts differences in ADHD and ODD growth, predictors were added to the LCAs from research question 1. Finally, to test research question 3 evaluating how ADHD and ODD co-vary over time, I conducted group-based dual trajectory analyses and cross-lagged models.

Final group-based trajectory models and cross-lagged models were tested for gender differences. To determine if there were gender differences in the distribution of boys and girls across classes, the final LCA for each domain was run with a binary gender variable covariate. To examine whether ADHD and ODD covary in different ways for boys and girls, I tested whether fixing parameters across gender in the crosslagged models constituted a significantly worse fit than a model in which parameters varied for boys and girls.

Past studies have indicated that $\mathrm{N} \geq 200$ is sufficient for distinguishing classes in group-based trajectory models with at least $80 \%$ power (Dziak et al., 2014, Nylund et al., 2007). Power analyses conducted in MPlus following a Monte Carlo approach (Thoemmes et al., 2010 indicated that a sample of 274 participants was sufficient to detect standardized effects in the cross-lagged model as small as approximately .11 with $80 \%$ power, a small to medium effect.

\subsubsection{Research Question 1: What are Common Trajectories of ADHD and ODD from} Ages 2 to 4?

Group-based trajectories across the five time points were evaluated for each symptom domain of ADHD (inattention, hyperactivity/impulsivity) as well as for ODD. 
Group-based trajectories are a type of latent class analysis (LCA) used to identify distinct growth patterns across time (e.g., Nagin, 1999). As outlined by Nagin (1999), in this model, each individual is assigned to a trajectory pattern based on posterior probabilities; for instance, a child with low symptom scores of ODD across time will show a high posterior probability of being in the low-symptom group and a low posterior probability (i.e., close to 0 ) of being in a high or increasing symptom group. Individuals are assigned to the trajectory group for which they have the highest posterior probability. In order to determine the best model fit, the Bayesian Information Criteria (BIC) and the Akaike Information Criteria (AIC) can be compared for nested and non-nested model; smaller BIC (e.g., Nagin, 1999, Huijbregts et al., 2007) and AIC (Busemeyer \& Diederich, 2014) are better. In the present study, trajectory models containing 3 and 4 classes were tested. I examined whether a quadratic term was significant and if models with quadratic growth showed better fit than a linear model. Model fit was compared using the BIC and AIC for all models.

\subsubsection{Research Question 2: Does Toddler Temperament Distinguish among Trajectory Groups?}

To address how children's temperament predicts growth of ADHD and ODD, LCAs with temperament traits as predictors were run. A number of different approaches have been suggested for examining predictors of latent classes. A one-step approach in which predictors are entered at the same time as forming latent classes has been critiqued. In such a model, covariates influence the formation of classes (Li \& Harring, 2017), which Vermunt (2010) argues is counter to the typical statistical approach of building a model. The classical three-step analysis for examining such questions is a multinomial 
logistic regression in which class membership is treated as an observed variable. There is some debate in the literature as to the appropriateness of this method. For instance, Feingold et al. (2014) describe that the classical three-step analysis leads to underestimating relations between variables; modified 3-step approaches have therefore been suggested by others (e.g., Asparouhov \& Muthén, 2014a, 2014b). In contrast, Clark and Muthén (2009) compared several different LCA regression models and found that estimates from the classical three-step model are generally similar to other models as long as entropy is above .80 and a more stringent alpha is used. In the present study I used the group-based LCA results from research question 1 so the predictors were not impacting the formation of the classes (i.e., not a one-step approach). I used a modified three-step model that built on the results of research question 1. I used syntax from Asparahouv \& Muthén (2014b) for an automatic modified 3-step model in MPlus in which predictors/covariates are specified as auxiliary variables using R3STEP. Starting values from the original LCA were used so that identified classes could be compared and were not re-estimated each time a covariate was added, a problematic method (e.g., Vermunt, 2010).

For each of the three final LCA models specified from research question 1 (for ODD and each domain of ADHD), separate LCA with covariate analyses were conducted for each temperament variable. These models provide logit values for how the temperament trait changes the likelihood of membership in one class compared to the reference class. All pairwise comparisons are provided with each class serving as the reference group. Logit values were exponentiated to calculate odds ratios; as the target 
temperament trait increases by 1 unit, the odds of a child ending up in one class compared to the reference group increases by a factor of that odds ratio.

\subsubsection{Research Question 3: How do Symptom Trajectories of ADHD and ODD Covary?}

To test how ADHD and ODD covary over time, I first examined group-based dual trajectory models (Nagin \& Tremblay, 2001). These models build on group-based trajectory models (Nagin, 1999) to simultaneously estimate two related, but distinct constructs. For my purposes, I estimated ADHD (each domain separately) with ODD trajectories. In each model, the group-based trajectories for both disorders are estimated; parameters are estimated for each class, defining the shape of that trajectory (e.g., intercept, slope), and posterior probabilities are used to determine class membership for each disorder (same as in group-based trajectories). Within the model, these posterior probabilities are used to calculate conditional probabilities of being in one class depending on membership in each class of the other disorder. Analyses were run once per direction for conditional probabilities because they are order dependent in Mplus. For each class of one disorder, the sum of probabilities of being in each of the classes from the other disorder should be one (Nagin \& Odgers, 2010).

Next, I examined a cross-lagged model for each inattention and hyperactivity/impulsivity domain with ODD. Traditional cross-lagged models have been critiqued since they conflate within-subjects and between-subjects change and because time-invariant factors might account for the effects (e.g., Besemer et al., 2016; Hamaker et al., 2015). Hamaker et al. (2015) also argues that traditional cross-lagged models are problematic when traits are stable, because the autoregressive paths do not fully account for this stability. Newer cross-lagged models have been developed (e.g., Besemer et al., 
2016; Hamaker et al., 2015) in order to address this concern. However, these models typically remove between-subjects variance. That is, these newer models test whether children with elevated symptoms relative to their own mean would be at risk for elevated symptoms of the other disorder relative to their own mean. In that sense, they remove any potential time invariant covariates or confounds that might drive between-subjects effects, since everyone is their own control. In contrast, I was interested in whether children with elevated symptoms at one time point relative to peers would be at risk for elevated symptoms of the other disorder at a later time point, controlling for early symptoms. The traditional cross-lagged design certainly does not exclude the possibility of findings being driven by within-subjects variance. However, I was not interested in only examining within-subjects difference. Further, I am examining ADHD and ODD across a highly dynamic time period, so although these symptoms are expected to show some stability, their stability is not expected to be as high as more invariant traits (which would be problematic; Hamaker et al., 2015). Thus, I selected the traditional cross-lagged model. However, I also ran final models as the random intercepts cross-lagged panel model (e.g., Hamaker et al., 2015) in order to compare to the traditional model and note any differences. In this model, a latent factor for each construct is added, and each observed variable is centered before the cross-lagged and autoregressive paths are added (syntax from Hamaker, 2018).

First, I began each series of models by constructing a baseline model. This model contained only autoregressive paths for ADHD (one domain) and ODD across the 5 time points, with concurrent correlations at each time point between ADHD and ODD (at Times 2 through 5, these correlations represent correlated residuals). Next, an ADHD 
effects model added in paths regressing Times 2 through 5 of ODD symptoms on the previous time point of ADHD symptoms, with the reverse for the ODD effects model. Finally, in the bidirectional model, symptoms of each ADHD and ODD from Times 2 through 5 were regressed on the previous time point of the other symptom domain. In all models, correlated errors with non-adjacent time points of the same symptom domain were estimated. Models with constrained paths across time were compared to models with free paths across time using chi-square difference tests. To determine the best model for each pair of factors, chi-square difference tests were used to examine whether each model was a significant improvement in fit over the baseline model, and whether the bidirectional model was an improvement over either of the unidirectional models. Further, model fit was assessed using: model $\chi^{2}\left(\chi^{2} / \mathrm{df}\right.$ below 2.0 is best, but up to 5.0 can indicate acceptable fit; Hooper et al., 2008), the Comparative Fit Index (CFI > .90 indicates acceptable fit, $\geq .95$ indicates better fit; Hooper et al., 2008; Hu \& Bentler, 1999), the root mean square error of approximation (RMSEA $<.08$ is acceptable fit, $<.06$ is better fit; Hooper et al., 2008; Hu \& Bentler, 1999), and Standardized Root Mean Square Residual (SRMR <.08 is acceptable, $<.05$ is better fit; Hooper et al., 2008; Hu \& Bentler, 1999), as well as examining the AIC and BIC (smaller is better). 


\section{CHAPTER 3}

\section{RESULTS}

\subsection{Descriptive Statistics}

Mean symptom counts and Likert-scale ratings, as well as skewness and kurtosis coefficients for ADHD and ODD at each time point are presented in Table 1. Outliers that were above or below 3.29 SDs from the mean (Tabachnick \& Fidell, 2013) were identified. Five participants were outliers in their total symptom counts of ODD. Six participants were outliers in their rating of inattention. Analyses were conducted both with and without these outliers. Results were mostly similar when repeated with and without these outliers, so final analyses included all participants. Any changes when outliers were excluded are noted as footnotes.

Independent samples $t$-tests were used to compare each symptom domain for boys and girls. There were no significant gender differences in mean levels of hyperactivity at Time 1, Time 2, Time 3, or Time 4 ( $p$ values from .082 to .559); there was a significant difference at Time 5 such that boys $(M=2.90, S D=2.54)$ showed more symptoms than girls $(M=2.00, S D=2.43), t(235)=2.08, p=.040$. For inattention, there were no significant gender differences at Time 1, Time 2, Time 4, or Time 5 ( $p$ s from .149 to $.173)$, but there was a significant difference at Time 3 such that boys $(M=1.78, S D=$

2.11) showed more symptoms than girls $(M=1.11, S D=1.69), t(160)=2.11, p=.037$. For ODD, there were no significant gender differences in symptoms at Time 1, Time 2, Time 3, or Time 4 ( $p$ s from .525 to .839 ), but there was a significant difference at Time 5 such that boys $(M=1.68, S D=2.13)$ showed more symptoms than girls $(M=0.96, S D=$ 1.92), $t(134)=2.00, p=.048$. 


\subsection{Research Question 1: What are Common Trajectories of ADHD and ODD from}

\section{Ages 2 to 4?}

\subsubsection{Hyperactivity/Impulsivity}

Group-based trajectory models with three classes and four classes were tested with both linear and quadratic growth (see Table 2). In each model, slope and quadratic term variances were set to 0 and random starts were increased to 500, with 50 iterations, in order to improve model estimation. The AIC and BIC values were compared for each model. The AIC was smallest for the four-class quadratic model, but the BIC was smallest for the three-class quadratic model and next smallest for the four-class quadratic model. In the four-class quadratic model, three of the four classes showed significant quadratic growth; the one class without quadratic growth was a stable low-symptom group, which we expected based on theory. In the three-class model, an improving group was not present. Thus, since the models were similar in fit, the four-class quadratic model was selected as the final model for hyperactivity/impulsivity since it included a wider range of developmental trajectories that toddlers might show (see Figure 1, Table 3). Overall, there was a high symptoms group, a low stable group, a declining symptoms group, and a moderate-increasing symptoms group. Almost half (47.62\%) of children in our sample were in the low-stable group, with a small number of symptoms across time and no significant rate of change. About a fourth of children $(27.84 \%)$ in our sample were in a high-symptoms group; this group showed negative quadratic growth, began with about 5 symptoms on average and ended with about 4.5 symptoms on average. A small portion of children $(8.79 \%)$ showed increasing quadratic growth; they began with a small-moderate number of symptoms (about 3 out of 9 symptoms) and increased in their 
symptoms as well as their rate of change of symptoms over time (moderate-increasing group). Finally, a small group of children (15.75\%) were a "terrible twos" group such that, on average, they began with a high number of symptoms, but decreased to have few symptoms over time, with rate of change slowing down over time. Note that of the 51 children who had a clinical level of symptoms of hyperactivity/impulsivity at Time 1 (i.e., six or more symptoms), $64.7 \%$ were in the high group, $27.5 \%$ were in the declining group, and $7.8 \%$ were in the moderately increasing group. Conversely, $43 \%$ of children in the high class of hyperactivity/impulsivity showed a clinical level of symptoms at Time 1.

An LCA with covariates (modified version; Asparahouv \& Muthén, 2014b) was conducted to test gender differences in the final trajectory. There was no significant difference between boys' and girls' likelihood of being in one class relative to another ( $p$ s from .172 to .876 ), suggesting that there was no significantly greater risk of chronic symptoms associated with one gender. That said, we cannot rule out the possibility that there are gender differences in growth. It is possible that while the overall pattern for children includes these four specific classes, gender-specific classes might be present. However, I was underpowered to detect effects with the smaller $n$ per gender.

\subsubsection{Inattention}

Group-based trajectory models with three classes and four classes were tested with both linear and quadratic growth (see Table 2). The AIC and BIC values were compared for each model. The AIC and BIC were smallest for the four-class quadratic model. In the four-class quadratic model, three of the four classes showed significant quadratic growth. Thus, the four-class quadratic model was selected as the final 
inattention model (see Figure 2 and Table 3). Overall, children were in a low-declining symptoms group, moderate-fast-increasing group, moderate-slow-increasing group, or high-moderate group. The majority of children $(69.96 \%)$ in the sample were in the lowdeclining symptoms group, with slightly positive quadratic growth such that symptoms were declining at a decreasing rate. A very small group of children (4.03\%) in the sample started with a moderate number of symptoms and showed negative quadratic growth; that is, they showed a steep increase in symptoms to a clinical level, which flattened in growth over time (moderate-fast-increasing group). Similarly, another small group of children (14.29\%) started with a moderate number of symptoms, but these children showed positive quadratic growth; that this, initially there was little growth but later they steeply increased in symptoms towards a clinical level of 6 symptoms (moderate-slowincreasing group). Finally, $11.72 \%$ of children started with high symptoms (around 5 symptoms) and showed a slight decline over time with no change in rate (no quadratic growth) to a moderate level (about 3-4 symptoms). Note that of the 25 children who had a clinical level of symptoms of inattention at Time 1 (i.e., six or more symptoms), 56\% were in the high group, $12 \%$ were in the moderate-fast-increasing group, $24 \%$ were in the moderate-slow-increasing group, and $8 \%$ were in the low group. Conversely, $44 \%$ of children in the high class of inattention showed a clinical level of symptoms at Time 1.

An LCA with covariates was conducted to test gender differences in the final trajectory. There was no significant difference between boys' and girls' likelihood of being in one class relative to any other ( $p$ s from .130 to .992), suggesting that there was no significantly greater risk of chronic symptoms associated with one gender.

\subsubsection{ODD}


Group-based trajectory models with three classes and four classes were tested with both linear and quadratic growth (see Table 2). The AIC and BIC values were compared for each model. The AIC was smallest for the four-class quadratic model (next highest was the four-class linear model), but the BIC was smallest for the four-class linear model (next highest was the four-class quadratic model). In the four-class quadratic model, two of the four classes showed significant quadratic growth, so I selected the quadratic model as the final ODD model (see Figure 3 and Table 3). Overall, children were in a low-stable group, moderate group, low-increasing group, or high-increasing group. The majority of children (75.82\%) were in the low-stable symptoms group, starting with 1 symptom on average and showing little change over time. A very small minority of children (2.93\%) started with about 1 symptom and increased with positive quadratic growth such that they showed more rapid growth over time until they had high symptoms by Time 5 (low-increasing group). A small group of children (6.23\%) started with a moderate-high number of symptoms (on average, 4 out of 8 symptoms) and showed increasing symptoms consistent with quadratic growth such that the rate of growth slowed down over time as children reached a high level of symptoms (highincreasing group). Finally, $15.02 \%$ of children showed a moderate level of symptoms over time (moderate group), starting around 2 symptoms and ending around 3 symptoms). Note that of the 30 children who had a clinical level of symptoms of ODD at Time 1 (i.e., four or more symptoms), $40 \%$ were in the low-stable group (in contrast to $81 \%$ of children who did not have clinical levels of symptoms of ODD at Time 1), $11 \%$ were in the high-increasing group, and 7\% were in the moderate group. Conversely, $65 \%$ of children in the high class of ODD showed a clinical level of symptoms at Time 1. 
An LCA with covariates was conducted to test gender differences in the final trajectory. There was no significant difference between boys' and girls' likelihood of being in one class relative to any other ( $p$ s from .201 to .900 ), suggesting that there was no significantly greater risk of chronic symptoms associated with one gender.

\subsection{Research Question 2: Does Toddlerhood Temperament Distinguish among Trajectory Groups?}

The final group-based trajectory model for each symptom domain was used to evaluate how temperament traits (effortful control, surgency, and negative affect) predict trajectories. Starting values from the final trajectory of ADHD domains and ODD were used so that trajectories did not change with the addition of covariates. This model produces a series of pairwise significance test comparing the level of each covariate for children in one class compared to the reference class; $p$ values from logits are reported and exponentiated logit parameters were calculated as odds ratios. See Table 4 for a summary of results.

\subsubsection{Hyperactivity/Impulsivity}

The final four-class quadratic model described above was used to evaluate how surgency, effortful control, and negative affect each predict differences between the four hyperactive/impulsive trajectories. Greater surgency was associated with higher odds of being in the high group compared to the low group, the moderate-increasing group compared to the low group, and the declining group compared to the low group. Greater effortful control predicted higher odds of being in the low group compared to the high group, the low group compared to the declining group, and the moderate-increasing 
group compared to the high group. Greater negative affect predicted higher odds of being in the high symptoms group compared to the low symptoms group.

\subsubsection{Inattention}

The final four-class quadratic model described above was used to evaluate how surgency, effortful control, and negative affect each predict differences between the four inattention trajectories. Greater surgency was associated with higher odds of membership in the high-moderate class compared to the low-declining class and the moderate-fastincreasing compared to the low class. Greater effortful control was associated with higher odds of membership in the low class compared to each the high-moderate class and the moderate-slow-increasing class. Greater negative affect predicted higher odds of membership in the high-moderate compared to the low class and the moderate-slowincreasing compared to the low class.

\subsubsection{ODD}

The final four-class quadratic model described above was used to evaluate how surgency, effortful control, and negative affect each predict differences between the four ODD trajectories. Greater surgency was associated with higher odds of membership in the high-increasing group compared the low-stable group and the moderate group compared to the low-stable group. Higher effortful control predicted greater odds of being in the low stable group compared to the moderate group and the high-increasing group. Higher negative affect predicted greater odds of membership in the high increasing group compared to the low-stable group and the moderate group compared to the low-stable group. 


\subsection{Research Question 3: How do Symptom Trajectories of ADHD and ODD}

\section{Covary?}

\subsubsection{Dual Trajectories}

Group-based dual trajectories were examined to test the conditional probability of being in one class given membership in another class (see Table 5). Low trajectories were linked. Children in the low hyperactivity/impulsivity class had a high probability of membership in the low ODD class (.867); conversely, children in the low ODD class had a high probability of membership in the low hyperactivity/impulsivity class $(\mathrm{P}=.672)$. High trajectories were also linked. Children in the high-increasing ODD class had a high probability of membership in the high hyperactivity/impulsivity class $(\mathrm{P}=.878)$. Conversely, children in the high hyperactivity/impulsivity group had the highest probability of being in the moderate ODD class $(\mathrm{P}=.559)$, followed by the highincreasing ODD class $(\mathrm{P}=.269)$. Similarly, children in the moderate ODD class had the highest probability of being in the high hyperactivity/impulsivity class $(\mathrm{P}=.505)$ compared to other hyperactivity/impulsivity classes. Increasing classes also were linked; children in the low-increasing ODD class had a high probability of being in the moderateincreasing hyperactivity/impulsivity class $(\mathrm{P}=.604)$. However, the converse was lower;

the moderate-increasing hyperactivity/impulsivity class had a similar probability of being in the low-increasing ODD class $(\mathrm{P}=.335)$ as in the low ODD class $(\mathrm{P}=.365)$ or the moderate ODD class $(\mathrm{P}=.300)$. Finally, children in the declining hyperactivity/impulsivity class had the highest probability of membership in the low ODD class $(\mathrm{P}=.685)$. 
For inattention and ODD, low trajectories were linked. Children in the lowdeclining inattention group had a high probability of being in the low ODD group $(\mathrm{P}=$ $.834)$ and vice versa $(\mathrm{P}=.878)$. High levels were also linked. Children in the high inattention class were most likely to be in the moderate ODD class $(\mathrm{P}=.620)$ followed by the high-increasing ODD class $(\mathrm{P}=.211)$. Conversely, children in the high-increasing ODD class were most likely to be in the high $(\mathrm{P}=.352)$ or moderate-slow-increasing $(\mathrm{P}=$ .335) inattention classes. Moderate levels were linked as well. Children in the moderateslow-increasing inattention class were most likely to be in the moderate ODD class $(\mathrm{P}=$ .482), although children in the moderate ODD class were most likely to be in the moderate-fast-increasing inattention class $(\mathrm{P}=.338)$.

For inattention and hyperactivity/impulsivity (see Table 6), low trajectories were linked. Children in the low hyperactivity/impulsivity class had a high probability of membership in the low inattention class $(\mathrm{P}=.965)$, as well as vice versa $(\mathrm{P}=.920)$. Children in the moderate-stable hyperactivity/impulsivity class had high probabilities of being in the moderate $(\mathrm{P}=.332)$ or low inattention groups $(\mathrm{P}=.466)$. Conversely, children in the moderate inattention class had a high likelihood of membership in the moderate-stable hyperactivity/impulsivity class $(\mathrm{P}=.603)$. Results for high groups were linked as well. Children in the high inattention group had a high probability of membership in the hyperactivity/impulsivity group $(\mathrm{P}=.920)$. The converse probability was lower $(\mathrm{P}=.349)$, and children in the high hyperactivity/impulsivity group were also likely to be in the moderate inattention group $(\mathrm{P}=.366)$. Finally, children in the declining hyperactivity/impulsivity class had a high probability of being in the moderate-slowincreasing inattention class $(\mathrm{P}=.739)$. Conversely, children in the moderate-slow- 
increasing group were most likely to be in the declining symptoms group $(\mathrm{P}=.402)$, but also were likely to be in the moderate-stable $(\mathrm{P}=.316)$ or high $(\mathrm{P}=.282)$ hyperactivity groups.

\subsubsection{Hyperactivity and ODD Cross-Lagged Models}

The baseline model showed that hyperactivity and ODD at each time point predicted their respective symptom domain at the following time point (all $p s<.001$ ). Further, ODD and hyperactivity at Time 1 were highly related, and hyperactivity and ODD residuals within each later time point were highly related (all $p s<.001$ ). This model showed adequate CFI (.94) and $\chi^{2} / \mathrm{df}=3.50$, but other fit statistics were not adequate $(\mathrm{RMSEA}=.10, \mathrm{SRMR}=.16, \mathrm{TLI}=.87)$. A model containing fixed paths from hyperactivity to ODD that were set to be equal across time was not a significantly worse fit than a free model $\left(\Delta \chi^{2}(3)=3.14, p=.371\right)$ and was thus selected as the best hyperactive effects model. In this model, all hyperactivity effects were significant $(p<$ .001). This model was a significantly better fit than the baseline model $\left(\Delta \chi^{2}(1)=35.92, p\right.$ $<.001)$ and showed adequate RSMEA (.05), SRMR (.07), CFI (.98), TLI (.96), and $\chi^{2} / \mathrm{df}$ (1.80). A model in which paths from ODD to hyperactivity were fixed across time was not a significantly worse fit than a free model $\left(\Delta \chi^{2}(3)=2.94, p=.401\right)$ and was thus selected as the best ODD effects model. In this model, ODD significantly predicted hyperactivity at all time points (standardized $p s$ from .004 to .007). This model was a significantly better fit than the baseline model $\left(\Delta \chi^{2}(1)=8.19, p=.004\right)$ and showed adequate CFI (.95) and $\chi^{2} / \mathrm{df}(3.26)$; however, several fit statistics were not adequate, including SRMR (.13), RMSEA (.09), and TLI (.88). The bidirectional model, with fixed cross-lagged paths across time for ODD to hyperactivity and for hyperactivity to ODD, 
provided a significantly better fit than the baseline model $(p<.001)$. In this model, hyperactivity predicted ODD at each subsequent time point, but ODD did not predict hyperactivity at any time point. The bidirectional model was not a significantly better fit than hyperactive effects model $\left(\Delta \chi^{2}(1)=2.66, p=.103\right)$, but was a better fit than the oppositionality effects model $\left(\Delta \chi^{2}(1)=30.39, p<.001\right)$. Since there were no significant ODD effects in the bidirectional model and this model did not improve fit over the hyperactive model, the simpler hyperactive effects model was chosen as a final model ${ }^{3}$, with paths fixed across time (see Figure 4).

The final model was tested for gender invariance by comparing a model in which cross-lagged paths from hyperactivity to ODD were set to be equal for boys and girls to one where these paths were free. The free model did not significantly improve the model $\left(\Delta \chi^{2}(1)=.114, p=.736\right)$. Note that when additional paths were also fixed (e.g., autoregressive paths, correlation between hyperactivity and ODD symptoms at each time point), there still was not significant improvement with free paths. Thus, there was no evidence that these relations were significantly different for boys and girls.

\subsubsection{Inattention and ODD Cross-Lagged Models}

The baseline model showed that inattention and ODD at each time point predicted their respective symptom domain at the following time point. Further, ODD and inattention at Time 1 and residuals at Time 2, 3, 4, and 5 were all positively related (all $p s$ $<.001)$. This model showed adequate CFI (.92) and $\chi^{2} / \mathrm{df}=3.93$, but other fit statistics

\footnotetext{
${ }^{3}$ The final hyperactive effects model with fixed paths to ODD was tested as a randomintercepts cross-lagged panel model to remove between-subjects invariance. No crosslagged paths were significant $(p=.161)$. Of note, cross-lagged paths in a bidirectional model were also not significant in either direction $(p s>.150)$.
} 
were not adequate $(\mathrm{RMSEA}=.10, \mathrm{SRMR}=.14, \mathrm{TLI}=.82)$. A model containing fixed paths from inattention to ODD set to be equal across time was a significantly worse fit than a free model $\left(\Delta \chi^{2}(3)=10.07, p=.018\right)$ and thus not selected as the best inattention effects model. The best inattention model, with paths free across time from inattention to ODD, was a significantly better fit than the baseline model $\left(\Delta \chi^{2}(4)=22.03, p<.001\right)$ and showed adequate CFI (.94) and $\chi^{2} / \mathrm{df}$ (3.53), but other fit statistics were not adequate $(\mathrm{TLI}=.85, \mathrm{SRMR}=.09$, and RMSEA $=.10)$. In this model, inattention only predicted ODD symptoms from T3 to T4 $(p<.001)$. A model in which paths from ODD to inattention were fixed was not a significantly worse fit than a free model $\left(\Delta \chi^{2}(3)=6.29\right.$, $p=.098)$. Thus, the best ODD effects model had fixed paths across time from ODD to inattention. In this model, ODD significantly predicted inattention at all time points (standardized ps from .010 to .014). This model was a significantly better fit than the baseline model $\left(\Delta \chi^{2}(1)=6.70, p=.001\right)$ and showed adequate CFI (.93) and $\chi^{2} / \mathrm{df}$ (3.78), but other fit statistics were not adequate $(\mathrm{TLI}=.83, \mathrm{RMSEA}=.10, \mathrm{SRMR}=.12)$. The bidirectional model, with fixed paths across time for ODD and free paths for inattention, was a significantly better fit than the baseline model $\left(\Delta \chi^{2}(5)=25.56, p<\right.$ .001). The model showed adequate CFI (.95), SRMR (.08), and $\chi^{2} / \mathrm{df}(3.53)$, but TLI (.82) and RMSEA (.10) were not adequate. In this model, inattention predicted ODD from T3 to T4 only $(p<.001)$ and ODD was trending to predict inattention at each time point $(p=.068) .{ }^{4}$ The bidirectional model was not a significantly better fit than

\footnotetext{
${ }^{4}$ Note that when outliers were removed, this model showed that ODD predicted inattention at each time point $(p=.001)$, whereas inattention was trending to predict or did not significantly predict ODD at each time point ( $p$ s from .094 to .953).
} 
inattention effects model $\left(\Delta \chi^{2}(1)=3.53, p=.060\right)$, although this was a trend. However, the bidirectional model was a significantly better fit than the ODD effects model ( $\Delta \chi^{2}(4)$ $=18.86, p<.001)$. This bidirectional model was chosen as the final model for inattention and ODD (see Figure 5). ${ }^{5}$

The final model was tested for gender invariance by comparing a model in which cross-lagged paths from inattention to ODD and from ODD to inattention were set to be equal for boys and girls to one where these paths were free. The free model did not significantly improve the model $\left(\Delta \chi^{2}(5)=1.54, p=.908\right)$. Note that when additional paths were also fixed (e.g., autoregressive paths, correlation between symptoms at each time point), there still was not significant improvement with free paths. Thus, there was no evidence for significant gender differences in these associations.

\footnotetext{
${ }^{5}$ The final bidirectional effects model with fixed paths from ODD to inattention and free paths from inattention to ODD was tested as a random-intercepts cross-lagged panel model to remove between-subjects invariance. In this model, inattention predicted ODD from T3 to T4 $(p=.044)$, as in the traditional model. ODD did not predict inattention at any time point $(p s=.226)$.
} 


\section{CHAPTER 4}

\section{DISCUSSION}

This study examined the development of ADHD and ODD symptoms from ages 2 to 4 in a community sample. I examined common trajectories of each disorder, temperament predictors of trajectories, and how ADHD and ODD co-develop. As expected, group-based trajectories showed that, although the majority of children had low symptoms over time, a small portion of children showed elevated, sustained levels of ADHD and ODD, and some children showed increasing symptoms. There was also evidence of a small portion of children who had a high initial level of hyperactivity/impulsivity symptoms and then declined to low symptoms, suggestive of a “terrible twos" phase; this was not evident for inattention or ODD. Temperament traits, including high negative affect, high surgency, and low effortful control, increased the likelihood that children would be in high or increasing symptom trajectories. Finally, as expected, children in high trajectories of symptoms of one disorder were more likely to be in high trajectories of the other, and ADHD was more predictive of ODD than vice versa. Overall, findings suggest that there are toddlers at risk for continued high symptoms of ADHD and ODD, especially children with certain temperament traits. Further, ADHD symptoms at an early age (particularly the hyperactive domain) seem to predispose children to developing ODD symptoms later. Findings have implications for early identification and intervention for externalizing symptoms.

\subsection{What are Common Trajectories of DSM Symptoms of ADHD and ODD from} Ages 2 to 4?

\subsubsection{Hyperactivity/Impulsivity}


The best model for hyperactive/impulsive symptoms showed four distinct growth patterns, some with quadratic growth. About half of the children were in a low stable symptoms group from ages 2 to 4 . A substantial minority of children (about a quarter) were in a high symptoms group, about half of whom showed a clinical level of symptoms at Time 1. A small number of children started with a moderate number of symptoms and showed increasing growth, and a small number of children were in a declining group, starting with a high number of symptoms and decreasing in their symptoms to a low level. Similar to past studies (e.g., Galéra et al., 2011, Salla et al., 2016; Shaw et al., 2005), there is a group of children for whom high symptoms at age 2 do not appear to be a phase. Past studies have varied in the rates of children who show high levels of symptoms over time, and findings from the present study were on the higher end, with about a quarter of children in the high stable group. However, there was also a group of children who did seem to grow out of their heightened symptoms at age 2, which only some past studies have identified (e.g., Shaw et al., 2005). It is possible that by examining a wide range of symptoms in a more national sample, we were able to elucidate an important growth trajectory that some studies may have missed. Importantly, other symptom domains examined in the present study (inattention and ODD) did not show such a clear declining symptoms group, suggesting that the normative increase in externalizing behaviors in toddlerhood (i.e., "terrible twos") may be best captured by hyperactive/impulsive symptoms.

\subsubsection{Inattention}

The best model of inattention symptoms showed four distinct growth patterns, several with quadratic growth. The majority of children (70\%) were in a low symptoms 
group, starting with few symptoms and declining slowly over time. Two groups of children showed moderate levels of symptoms at age two and increased over time; one group increased quickly early on and the other group increased slowly at first. Finally, a small group of children showed high-moderate symptoms (starting around 5 symptoms and ending around 3-4), about half of whom showed a clinical level of symptoms at Time 1. The fact that the majority of children showed few symptoms of inattention across toddler to preschool years was unsurprising given that inattention symptoms often emerge later than hyperactive/impulsive symptoms (e.g., Berger \& Nevo, 2011; Willoughby et al., 2012). Consistent with such findings, there were two groups of children who showed increasing inattentive symptoms over time. However, in contrast to expectations, there was no significantly declining symptoms group. Although the group starting with high symptoms declined slightly, this decline was not significant and not very large, suggesting less of a "terrible twos" decline compared to the hyperactivity/impulsivity domain. However, it is possible that I was underpowered to detect this effect. Importantly, the trajectory patterns for inattention were different from those of hyperactive/impulsive symptoms, underscoring the need to examine these domains separately.

The present study was an important extension of past studies that mostly examined the hyperactive/impulsive domain of ADHD and not the inattention domain. Trajectory studies that have examined the inattention domain (e.g., Galéra et al., 2011; Salla et al., 2016) have found three groups of low, moderate, and high levels of inattention, with some increasing growth over time. The present study similarly showed that most children are not in high symptom groups and that symptoms increase for some 
children. However, I found that a 4-class model was the best fit, compared to the 3-class model in previous studies. This difference in the number of classes might be attributable to measurement; the present study included 9 DSM-based symptoms of inattention whereas these past studies each used only three symptoms. Thus, I may have been able to identify more precise growth patterns, such as the fast and slow increasing groups. Additionally, using all 9 DSM-based symptoms allowed for comparison to clinical cutoffs (6 symptoms). For instance, I was able to identify that some children approach this clinical level later in preschool years, indicating that increasing symptoms might lead to problematic impairment.

\subsubsection{ODD}

The best model for ODD showed four distinct trajectories. The majority of children $(75 \%)$ were in a low-stable group, showing about 1 symptom over time. A small portion of children showed a stable, moderate level of symptoms over time. Another small group of children were in a high symptoms group, starting with about 4 symptoms and increasing rapidly at first to even higher symptoms, before growth slowed down; about two-thirds of children in this group started with a clinical level of symptoms at Time 1. Finally, the smallest group of children started with a low number of symptoms and increased, more quickly over time, to show a high number of symptoms by Time 5 . Findings in past studies with toddlers have similarly found low, medium, and high symptom groups, although specific studies show slight differences. For instance, Cote et al. (2006) and Shaw et al. (2005) each found high and low groups, and each found 1-2 moderate declining groups, whereas Huijbregts et al. (2007) found a low group as well as two groups of children with moderate or high levels. However, these studies did not 
utilize DSM-based criteria and they focused on physical aggression (Cote et al., 2006; Huijbregts et al., 2007) or a small number of general conduct problems (Shaw et al., 2005). The measure used in the current study reflected the diagnostic criteria for ODD and utilized more items than these previous studies, which may have been more sensitive to changes over time. That said, findings in the present study were similar to past studies in that most children were in a low symptom group and a small portion showed either high or moderate symptoms over time. In contrast to past studies, I also found a very small group of children who started with a low level of symptoms and rapidly increased to a high level. It is possible that using more items than in past studies allowed for greater sensitivity to detect more changes over time. These discrepancies across studies provide some rationale for examining subdomains of ODD symptoms in future studies (e.g., negative affect, antagonistic behavior) as these domains might show different growth patterns. Importantly, I had expected that there would be a declining ODD symptoms group, similar to past studies and consistent with theory about heightened oppositionality at age two. Surprisingly, there was not such a declining ODD symptoms group. As noted above, perhaps hyperactive/impulsive symptoms better capture the transient behavior difficulties that toddlers tend to show, while the presence of high levels of ODD behaviors (e.g., oppositionality, negative affect) compared to peers may be a more likely indication of developing psychopathology.

\subsubsection{Gender Differences in Growth}

The overall trajectories did not have a different proportion of boys and girls in each trajectory. Further, mean levels of symptoms were generally not significantly different at each time point. I expected stronger gender differences, with boys showing 
more propensity for high symptoms across the board. However, it is possible that gender differences widen as children age and are socialized into gender expectations. Relatedly, past studies have found consistent gender differences in older children for ADHD (e.g., DuPaul et al., 2016) and ODD (e.g., Demmer et al., 2017), whereas findings with younger children are mixed; some studies with younger children show few gender differences (e.g., Lavigne et al., 2009) and others show that boys have more severe symptoms (e.g., Baillargeon et al., 2007; McGoey et al., 2007) or are at risk for high symptoms trajectories (e.g., Romano et al., 2006) compared to girls. The present study somewhat supports findings that younger boys show more propensity for higher symptoms of both disorders, based on a handful of mean differences at later time points. Such emerging differences are consistent with theory that gender socialization effects are visible even in toddlers (e.g., Leaper \& Friedman, 2015). Overall though, the study found few gender differences in growth. That said, I was underpowered to examine trajectories separately for boys and girls, and it is possible that they may show different patterns of growth or numbers of classes.

Importantly, the fact that gender differences appear to widen as children age might reflect biases in referral patterns. For instance, there is some evidence that teachers are more likely to refer school-aged boys than girls with hyperactive/impulsive symptoms (e.g., Sciutto et al., 2004), perhaps related to finding these symptoms to be more problematic and disruptive than inattention symptoms (e.g., Coles et al., 2012). Inattention symptoms are more common in girls (e.g., Groenewald et al., 2009), so girls may be less likely to get referred if these symptoms are seen as less problematic. At the same time, girls that do show hyperactive/impulsive symptoms are rated as more 
impaired than boys (e.g., Coles et al., 2012), which suggests that teachers may expect more disruptive behavior from boys than girls. Overall, it is hard to determine if differences in ratings by gender reflect true underlying differences or biases in identifying impairment or referral. There are certainly real consequences if girls' symptoms are missed, such as losing access to treatment. Although only speculative, it is likely that bias factored into ratings in the present study, such as parents' differential expectations of children's behavior by gender. Future studies may want to examine the mechanisms behind these widening gender differences in ratings as children age.

\subsection{Does Toddler Temperament Distinguish Among Trajectory Groups?}

Temperament traits predicted the likelihood of children's membership in different classes of ADHD and ODD growth. Overall, as expected, high surgency and negative affect as well as low effortful control were risk factors for children following higher or increasing symptom trajectories. However, there was less distinction across domains than expected. All three temperament domains were risk factors for being in high or increasing trajectories of both domains of ADHD as well as ODD, relative to low symptom groups. Interestingly, temperament traits did not predict the likelihood of being in one class over another for classes that started with similar levels. For example, there were no significant differences for the high versus declining groups for hyperactivity/impulsivity. This pattern of results suggests that temperament traits might be more associated with level of symptoms early on (i.e., Time 1) rather than with changes over time.

Results were somewhat consistent with expectations, but I expected more specificity of risk from each temperament domain based on theory. Martel (2009) outlines several models suggesting that low effortful control is a risk factor specifically 
for inattention symptoms, high surgency and negative affect are risk factors for hyperactivity/impulsivity, and negative affect is a predictor for ODD. Present findings do show such linkages aligned with Martel's (2009) theoretical model. However, additional significant relations were also present that suggested less specificity of effects. Some past findings have similarly found that all of these temperament domains are risk factors for ADHD and ODD (e.g., Wichstrøm et al., 2018) and more generally for problem behavior (e.g., Stifter et al., 2008), with less domain specificity. However, these studies generally refer to risk of high levels of symptoms rather than risk for different symptom growth trajectories. Findings regarding temperament risk for growth trajectories have been mixed regarding the direction of effects. Some have found no relation between temperament and symptoms (e.g., Owens \& Shaw, 2003), whereas others have found that difficult temperament predicts declining symptoms (e.g., Miner \& Clarke-Stewart, 2008). Others have found that worsening effortful control predicted increasing ADHD and ODD symptoms (e.g., Atherton et al., 2019). A number of studies (e.g., Kochanska \& Kim, 2013) have also found that the effect of temperament on symptoms is moderated by environmental factors; the present study did not assess this theory directly. The direction of present findings is most aligned with Atherton and colleagues (2019) in that temperament traits like low effortful control predicted higher ADHD and ODD symptoms over time, however, this past study examined changes in temperament over time which I did not. Overall, there is a generally small body of literature on temperament risk for ADHD and ODD trajectories, and most studies with young children have used a handful of externalizing symptoms (e.g., Owens \& Shaw, 2003) rather than a 
broader spectrum of DSM-based symptoms. Thus, differences in measurement might account for some differences in study results.

There could be several reasons that these specific temperament traits are risk factors for high ADHD and ODD trajectories. One reason is related to the vulnerability model (Tacket, 2006) in that these traits might predispose children to developing or sustaining symptoms of psychopathology. Specifically, deficits in effortful control are related to top-down processing and cognitive control. Such ability to control one's own behavior and emotions is impaired in both of these disorders. Deficits in surgency and negative affect are more related to bottom-up processing, including reactive control in response to positive and negative emotions. Emotion dysregulation is a core deficit in both ADHD and ODD (e.g., Cavanagh et al., 2017; Graziano \& Garcia, 2016), and particularly in the hyperactivity/impulsivity domain of ADHD (e.g., Martel et al., 2009; Seymour et al., 2012). Some past studies suggest that the effects of difficult temperament might interact with factors like parenting or parent psychopathology to affect externalizing symptoms (e.g., Kochanska \& Kim, 2013; Owens \& Shaw, 2003). Second, there may be other variables that explain the relation, such as executive functioning. For instance, Tiego and colleagues (2020) found that executive function and effortful control were highly correlated and represented a "self-regulation" factor; this factor accounted for most of the observed variance in ADHD symptoms. In another study, executive dysfunction mediated the relation between early effortful control and later ADHD (e.g., Rabinovitz et al., 2016). Thus, there may be other variables that explain the relation between temperament and externalizing disorders, though more research might be needed to clarify this relation. Third, there may be more of a semantic shift as children age. That 
is, we may consider younger children to have more "temperament difficulties" whereas we identify the same symptoms in older children as symptoms of psychopathology. The fact that temperament difficulties at Time 1 were associated with high stable groups (i.e., high levels of symptoms at Time 1) suggests that there might be some overlap between these concepts.

\subsection{How do Symptom Trajectories of ADHD and ODD Co-vary?}

\subsubsection{Dual Trajectories}

In the present study, I found evidence that ADHD and ODD symptoms co-vary over time. Dual growth trajectories showed associations between trajectories of ODD and both domains of ADHD. Children in low trajectories of ODD were highly likely to be in low trajectories of each domain of ADHD, and vice versa. High symptoms were also linked, but the direction of risk showed some differences. Specifically, children with high-increasing ODD symptoms were most likely to be in high ADHD classes (both domains). Conversely, children in high ADHD classes (both domains) were most likely to be in the moderate ODD class, followed by the high-increasing ODD class. Findings were highly similar to past studies using dual trajectories with ADHD and ODD (e.g., Jester et al., 2005, 2008; Fontaine et al., 2008; Shaw et al., 2005) in that high groups were linked and low groups were linked. Present findings are also consistent with research on prevalence rates of comorbidities of these disorders, which are somewhat asymmetrical. Specifically, most children with ODD also have comorbid ADHD (e.g., Greene et al., 2002). In contrast, about 40-50\% of children with ADHD also have ODD, with slightly lower numbers for children who only have inattention symptoms (Elia et al., 2008; Reale et al., 2017). In present findings, children with high ODD were more likely to have high 
symptoms of both domains of ADHD than the reverse. That said, the pattern of conditional probabilities suggested that a substantial portion of children with high ADHD symptoms demonstrated high symptoms of ODD that worsened over time. These results were consistent with cross-lagged findings in the present study which showed that higher ADHD (particularly hyperactive/impulsive) symptoms predicted later ODD symptoms. The present findings add to a small body of literature suggesting trajectories are linked even in young children as symptoms first emerge (e.g., Shaw et al., 2005). In general, past studies with dual trajectories focused on older children and used more general measures of externalizing behaviors with a small number of items. The present study extends these findings to a larger number of DSM-based symptoms, beyond just a handful of externalizing symptoms like aggression.

Of note, trajectories of the two domains of ADHD were also linked such that children in the high group of one were likely to be in the high group of the other domain, and children in the low group of one domain were likely to be in the low domain of the other. Results were similar to past studies linking the two ADHD domains (e.g., Galéra et al., 2011). Interestingly, the majority of the children in the declining hyperactivity/impulsivity group were in the moderate-slow-increasing inattention group. Conversely, although children in the moderate-slow-increasing class were most likely to be in the declining group, they also were likely to be in the high or moderate-stable hyperactivity/impulsivity group. These findings are particularly important because only the hyperactivity/impulsivity domain showed a "terrible twos" declining group. It seems that children who decline in these symptoms over time are still at risk for developing inattention symptoms later in the preschool years. In fact, whether children "grow out" of 
early high symptoms of hyperactivity/impulsivity or continue to show high symptoms, they are at risk for increasing inattention, suggesting that toddlerhood heightened hyperactivity/impulsivity behavior (i.e., Time 1 level) rather than symptom growth is a risk factor for increasing inattention. In contrast, children who showed slowly increasing inattention symptoms were still most likely to grow out of hyperactivity/impulsivity symptoms, perhaps indicating that inattention is less of a risk factor for hyperactivity/impulsivity than vice versa.

\subsubsection{Cross-Lagged Effects}

I examined the direction of the effects between ADHD and ODD using a series of cross-lagged models. Hyperactivity/impulsivity predicted ODD symptoms at subsequent time points, but ODD did not significantly predict hyperactivity/impulsivity. These relations were not significantly different across time and not significantly different for boys and girls. Hyperactivity/impulsivity symptoms thus appeared to be a risk factor for ODD symptoms uniformly across this age period. In contrast, inattention and ODD showed bidirectional relations. Inattention predicted ODD symptoms, but only from Time 3 to Time 4, whereas ODD predicted inattention symptoms at all time points. As with hyperactivity/impulsivity, there were no significant gender differences in these effects. I also explored cross-lagged effects using a random intercepts cross-lagged panel model. This modified cross-lagged model removes between-subjects variance and might better account for stability of traits over time than using autoregressive paths (Hamaker et al., 2015). The three domains in the present study were all moderate to highly stable across time, so it possible that the autoregressive paths did not fully account for this stability in the traditional model. Results from the modified model showed non-significant cross- 
lagged paths. Although we can only cautiously interpret null findings, results suggest that perhaps the directional results we found in the traditional cross-lagged model are due to between-subjects effects. That is, children high in their hyperactivity/impulsivity at one time point relative to their peers are at risk for increased symptoms of ODD at the next time, relative to their peers. Of note, although ODD and both domains of ADHD were all fairly stable traits, hyperactivity/impulsivity showed the highest stability. It is possible that since hyperactivity/impulsivity symptoms were relatively more stable over time than ODD symptoms, it was more difficult for ODD to significantly predict hyperactivity/impulsivity than vice versa. The less stable trait is more often found to be predictive of the more stable trait than the other way around (see Anderson \& Kida, 1982). Thus, results should be interpreted with caution.

Findings do support the need to examine ADHD domains separately, particularly since there is much less research on the inattention domain compared to the hyperactive/impulsive domain. Similar to past cross-lagged literature (e.g., Burke et al., 2005; Burns \& Walsh, 2002), I found that hyperactivity/impulsivity symptoms confer risk of ODD symptoms. Results were consistent with dual trajectory models showing that children with high hyperactivity/impulsivity were likely to show increasing ODD symptoms. Consistent with past studies that found that inattention did not predict ODD symptoms (e.g., Burke et al., 2005), the inattention domain seemed to be less of a risk factor for ODD, although in present findings, inattention did predict ODD at one time point. ODD symptoms did predict inattention symptoms, similar to findings in older children (Burke et al., 2005) and younger children (e.g., Harvey et al., 2016). Of note, Harvey et al. (2016) found that only one subdomain of ODD (argumentative/defiance) 
predicted inattention, at only some time points, and only for boys; although I was not able to examine subdomains of ODD in the present study, it may be that one subdomain of symptoms was driving this effect and also might reveal gender differences. In addition, findings that inattention and ODD predicted one another mapped onto dual trajectory findings that children with quickly increasing inattention symptoms were likely to show high, increasing ODD symptoms and, conversely, that children with increasing ODD symptoms (low or high) were likely to show increasing inattention symptoms.

\subsubsection{Theory Underlying Co-development}

Although we cannot tease apart the exact mechanism for the observed effects, results map onto past theory. The fact that hyperactivity/impulsivity preceded ODD is aligned with theory that suggests ADHD leads to ODD via disruptions in family processes like parenting (Harvey et al., 2016; Kaiser et al., 2010). In fact, some past findings suggest that this directional relation is unique to hyperactivity/impulsivity (e.g., Nelson et al., 2019); present findings are somewhat aligned with this given that hyperactivity/impulsivity was more consistently predictive of ODD symptoms than inattention. Although there is less theoretical explanation in the literature for the relation between inattention and ODD, results suggest that these domains are linked. One possible mechanism is that hyperactivity/impulsivity symptoms increase risk of both inattention and ODD symptoms, thereby creating a bidirectional relation between inattention and ODD. Dual trajectory results showing that the two domains of ADHD are linked might support this idea. It is also possible that links between both domains of ADHD and ODD reflect underlying risk factors, such as genetic predisposition or temperament traits of surgency, effortful control, and negative affect, as observed in the present study. Some 
conceptual overlap could also account for this bidirectional relationship; for instance, children may appear to "not listen" in either a defiant manner or in an inattentive manner. Finally, it is also possible that there are specific directional causes between the two symptom domains. For example, disruptions in attentional control, particularly as children are entering preschool, might make children more vulnerable to negative affect and other ODD symptoms if they are less able to direct their attention away from negative stimuli. Conversely, strong emotional reactions characteristic of ODD might interfere with children's ability to focus or follow through on tasks (inattention symptoms). More research is needed to elucidate mechanisms behind the inattention-

ODD link. Further, although findings with school-age and adolescent children are more consistent in showing that hyperactivity/impulsivity predicts ODD, relations in younger children (e.g., Harvey et al., 2016) might be less clear-cut as symptoms and developmental changes at this age are highly dynamic.

\subsection{Summary of Findings}

Overall, findings suggest that some children who show a high level of symptoms in toddlerhood seem to be at risk for continued symptoms over early childhood years. That said, these early years do seem dynamic, with some children growing out of early high levels of hyperactive/impulsive symptoms and some children developing symptoms of ADHD or ODD later in the preschool years. Further, difficult temperament is associated with more chronic symptoms, pointing to possible early childhood indicators of psychopathology. However, more research is needed to examine other predictors that might better distinguish between children in transient phases versus at-risk for chronic hyperactive/impulsive symptoms. The study also sheds light on the co-development of 
ADHD and ODD, which are commonly comorbid. Children in high trajectories of one disorder were likely to be in high trajectories of the other, but there was some directionality to the co-development. Specifically, high hyperactive/impulsive symptoms seem to increase children's risk of developing more ODD symptoms over time, while relations between inattention and ODD were more bidirectional. Findings underscore the importance of examining separate domains of ADHD. In sum, for a portion of children, early signs of ADHD might indicate chronic difficulties and risk for increasing ODD symptoms.

\subsection{Limitations}

This study adds to our understanding of the early emergence of ADHD and ODD symptoms. That said, there were a number of limitations. First, the participants were drawn from a larger study that had high attrition. In order to assess my specific research questions, I only used participants who had completed at least one follow-up. Findings might be less generalizable because children who only completed Time 1 were excluded. Further, trajectory estimates might be less reliable since some children had only two timepoints. Second, the number of children in moderate or higher trajectory groups was smaller than the number of children in the low groups. Estimates of the higher trajectories may be less precise, and future studies may want to oversample for children in a clinical range to gain more accurate estimates of their growth. Third, I was underpowered to examine gender-specific trajectories. It may be that the pattern of growth is different for boys and girls, even if the gender proportions in the overall trajectories were not significantly different. Fourth, we do not know if one domain of ODD was driving findings. Given that subdomains have so few symptoms, results were highly unstable 
when I tried examining subdomains. Since past studies have identified that subdomains of ODD might show different patterns of results (e.g., Harvey et al., 2016), present results may have differed by ODD domain. Fifth, as described in the analytic plan section, crosslagged models have been critiqued for conflating within- and between-subjects effects and because the autoregressive paths might not fully account for the stability in traits (e.g., Hamaker et al., 2015). That said, I did compare results to a within-subjects only model to better tease apart between- and within-subjects effects. Sixth, the same rater completed all measures so shared method variance might contribute to some of the findings. Seventh, while using a national sample is more geographically representative than a local sample, there are other ways the sample may be less representative; for instance, families were mostly white and parents were more highly educated than national averages. Finally, an important part of assessing ADHD and ODD includes evaluating the level of impairment, and not simply whether the behavior is present. The present study only examined symptom count and did not make diagnoses. I do expect that high symptom levels would be more impairing for children and families, but this should be explicitly examined.

\subsection{Implications and Future Directions}

Findings shed some light on the potential for early identification and intervention in toddlerhood. Specifically, early signs of ADHD and ODD in toddlerhood are important to take seriously and might indicate chronic difficulties with these disorders. That said, some children do seem to grow out of a transient developmental phase characterized by high hyperactive/impulsive symptoms. It is important for future studies to examine risk factors that might distinguish between chronic and declining symptom groups, since I did 
not find support that temperament traits differentiate between such groups. Further, extending studies to a larger age range will allow us to understand the common course of trajectories beyond age 4 and link toddlerhood behavior to symptoms in middle childhood. In addition, across dual trajectory and cross-lagged findings, children with high hyperactive/impulsive symptoms seemed to be at risk for having high or increasing ODD symptoms. Thus, early interventions to address ADHD symptoms (particularly the hyperactive/impulsive domain) might reduce the likelihood of developing co-occurring ODD symptoms. There are a number of parent training interventions for addressing ADHD (especially more disruptive hyperactive/impulsive symptoms) in preschoolers (see Coates, Taylor, \& Sayal, 2016) and programs focused on working with parents of toddlers have found reductions in behavior problems (e.g., Hutchings et al., 2017; Niccols, 2009). By reducing early behavior difficulties, we might be able to mitigate the risk of children developing future co-occurring symptoms and having poorer outcomes. A particular challenge is that many early externalizing symptoms are written off as a developmental phase. The present study results suggest that some children with early high levels of symptoms are at risk for chronic symptomatology. As we develop more sensitive tools to identify children most at-risk, early screening of ADHD and ODD symptoms can hopefully become more commonplace. A long-term goal is for screenings to help medical and mental health professionals refer children who might benefit from early intervention. 
Figure 1

Hyperactivity/impulsivity Trajectories from Time 1 to Time 5

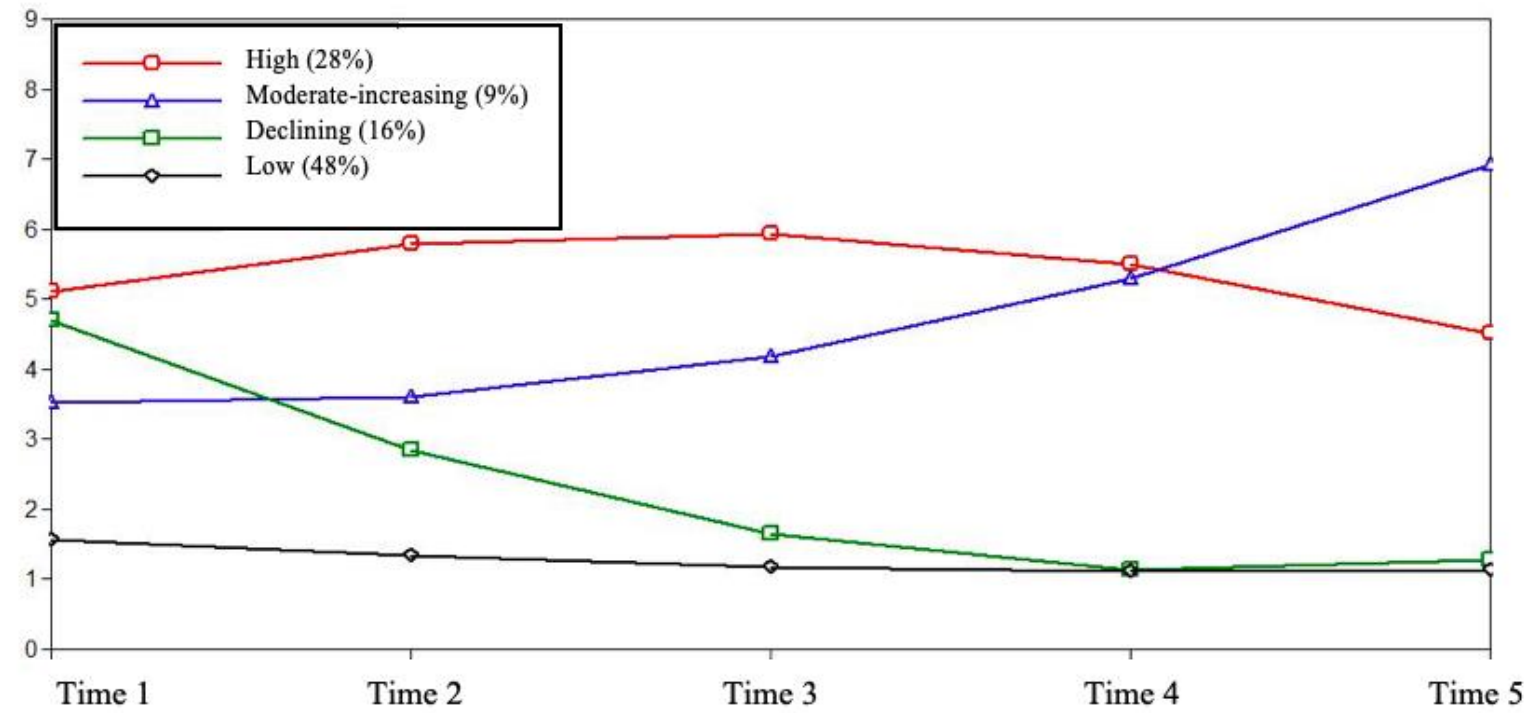


Figure 2

Inattention Trajectories from Time 1 to Time 5

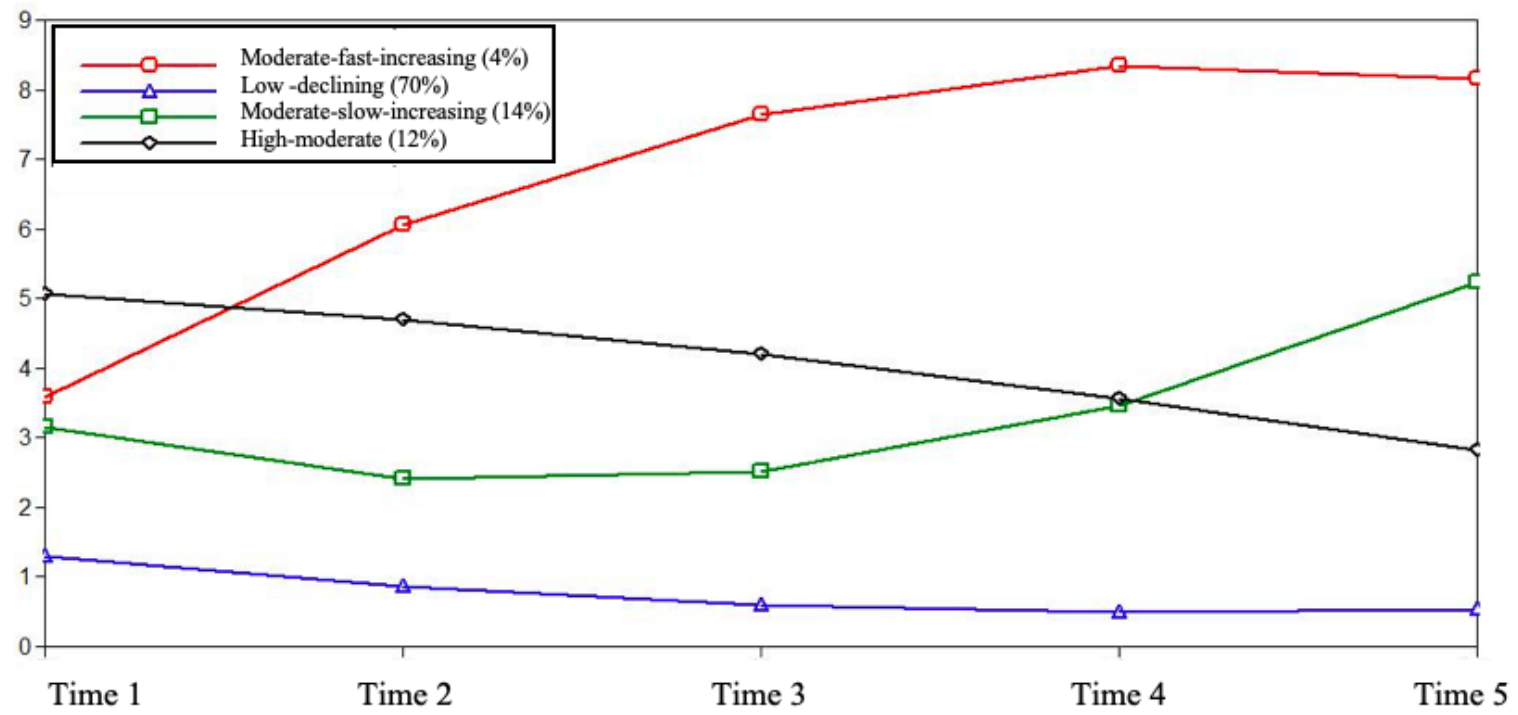


Figure 3

ODD Trajectories from Time 1 to Time 5

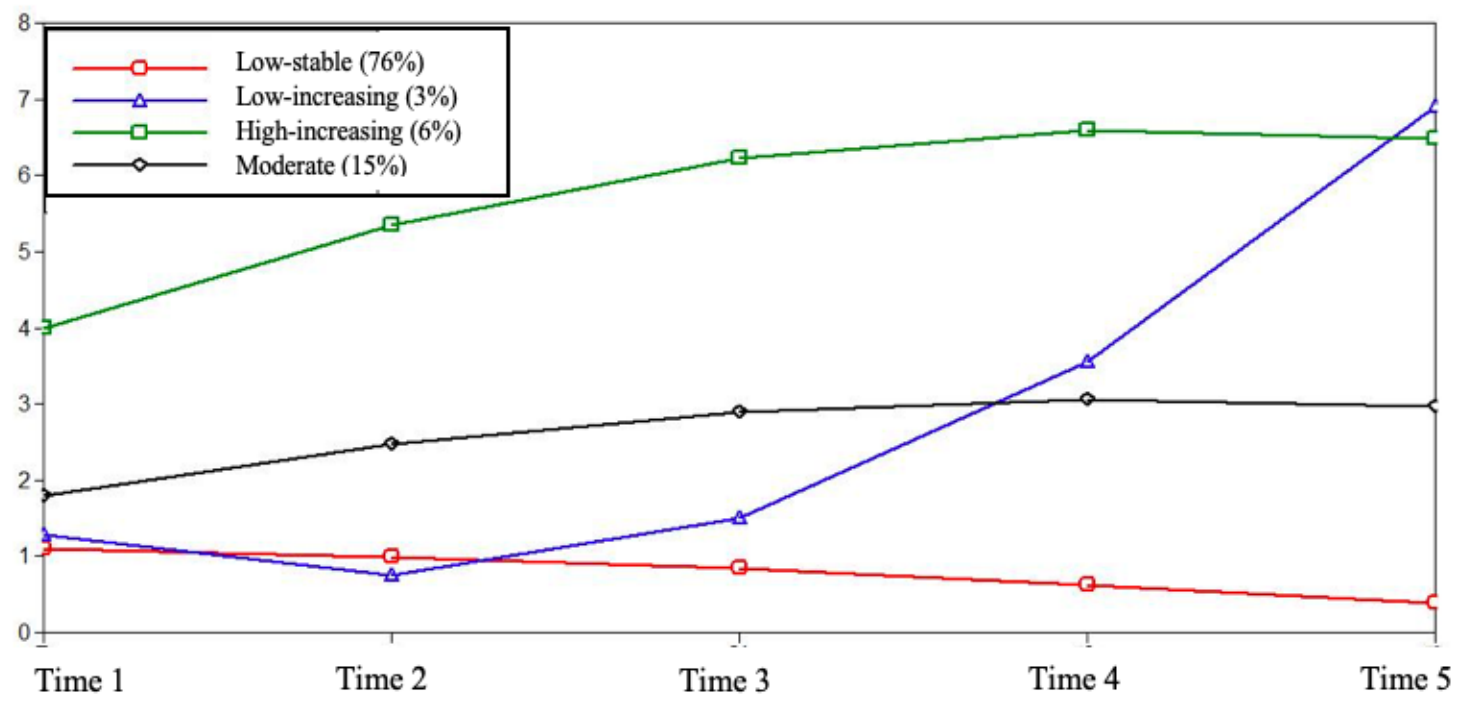




\section{Figure 4}

Hyperactive Effects Model between Hyperactivity and ODD, with Fixed paths Across time from Hyperactivity to ODD

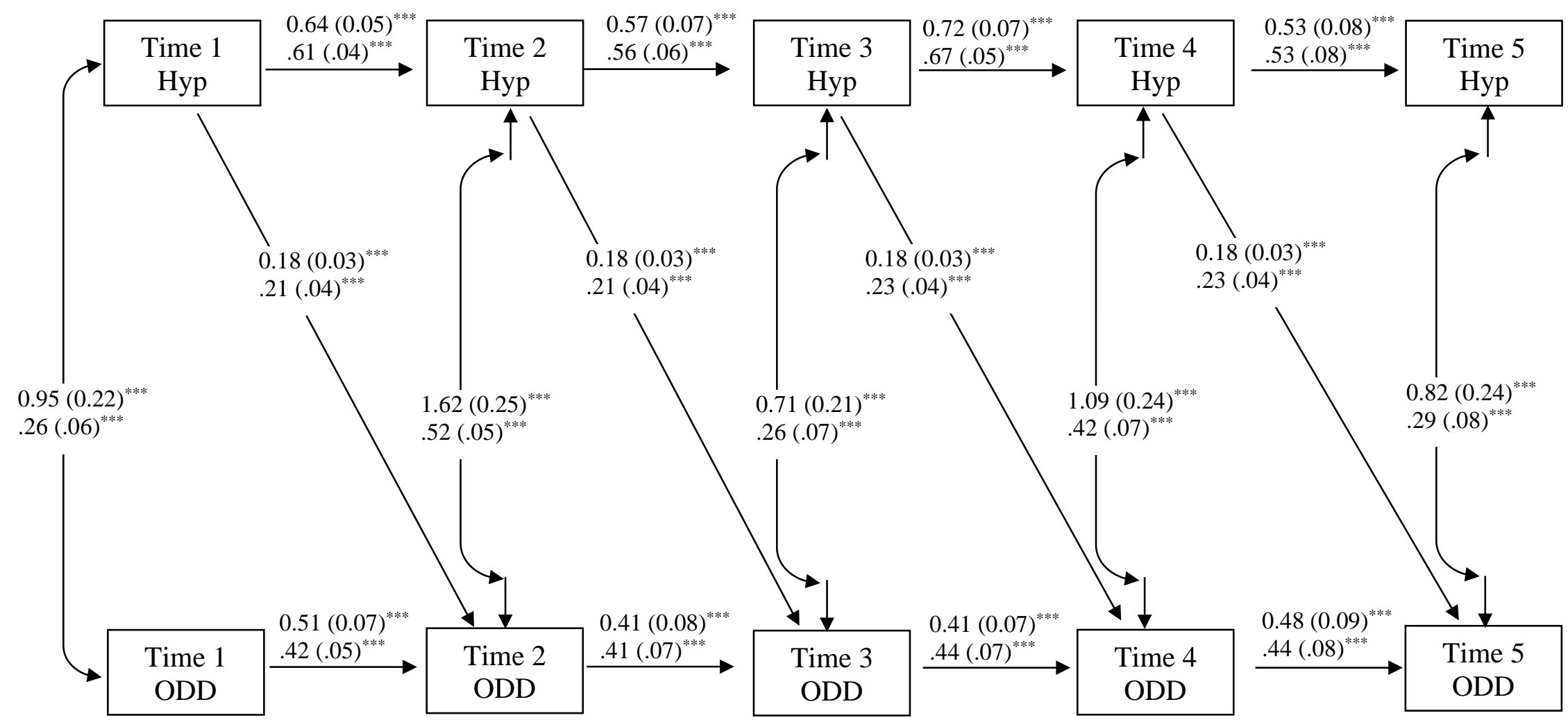

Note. Unstandardized results are listed above standardized results. Correlated errors for non-adjacent time points were estimated but not shown for ease of presentation. ODD = oppositional defiant disorder; Hyp = hyperactivity/impulsivity domain of ADHD. $* * * p<.001 ; * * p<.01 ; * p<.05$ 


\section{Figure 5}

Cross-lagged Model between Inattention and ODD, with Fixed Paths across Time from ODD to Inattention

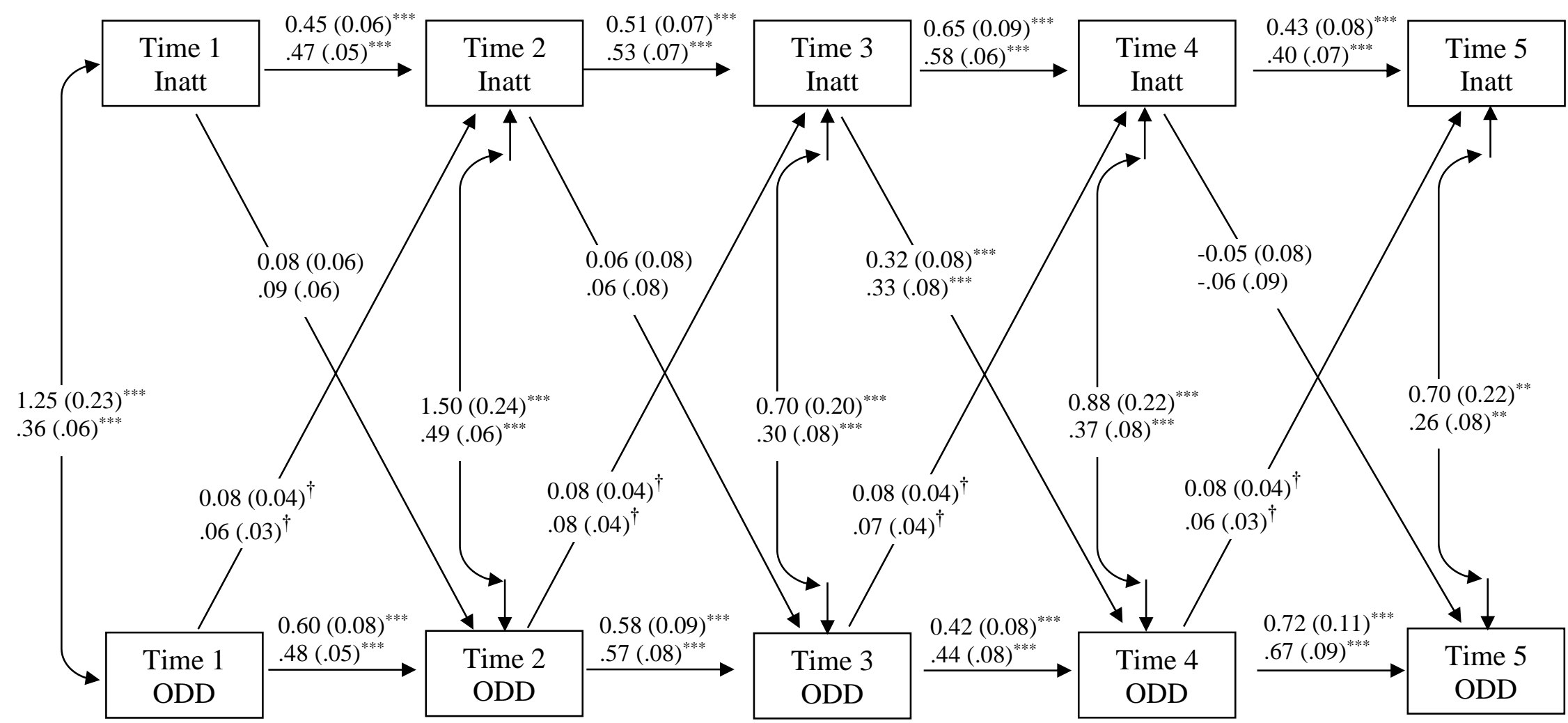

Note. Unstandardized results are listed above standardized results. Correlated errors for non-adjacent time points were estimated but not shown for ease of presentation. When outliers were removed, ODD predicted inattention at each time point $(p=.001)$, whereas inattention was trending to predict or did not significantly predict ODD at each time point ( $p$ s from .094 to .953). ODD = oppositional defiant disorder; Inatt $=$ inattention domain of ADHD.

*** $p<.001 ; * * p<.01 ; * p<.05 ; \dagger p<.10$ 
Table 1

ADHD and ODD Average Symptom Ratings and Symptom Counts

\begin{tabular}{|c|c|c|c|c|c|c|}
\hline & \multicolumn{2}{|c|}{ Average rating ( 0 to 3 ) } & \multicolumn{4}{|c|}{ Average symptom count } \\
\hline & $M$ & $S D$ & $M$ & $S D$ & Skew & Kurtosis \\
\hline \multicolumn{7}{|c|}{ Hyperactivity/ } \\
\hline \multicolumn{7}{|c|}{ Impulsivity } \\
\hline Time 1 & 1.24 & 0.59 & 3.23 & 2.28 & 0.25 & -0.91 \\
\hline Time 2 & 1.16 & 0.62 & 2.98 & 2.37 & 0.61 & -0.47 \\
\hline Time 3 & 1.12 & 0.63 & 2.70 & 2.49 & 0.60 & -0.82 \\
\hline Time 4 & 1.12 & 0.67 & 2.80 & 2.62 & 0.53 & -0.96 \\
\hline Time 5 & 1.08 & 0.67 & 2.53 & 2.53 & 0.95 & -0.03 \\
\hline \multicolumn{7}{|c|}{ Inattention } \\
\hline Time 1 & 1.03 & 0.58 & 2.20 & 2.20 & 0.95 & 0.23 \\
\hline Time 2 & 0.90 & 0.54 & 1.73 & 2.11 & 1.30 & 1.05 \\
\hline Time 3 & 0.86 & 0.53 & 1.52 & 1.98 & 1.61 & 2.61 \\
\hline Time 4 & 0.87 & 0.57 & 1.62 & 2.15 & 1.61 & 2.30 \\
\hline Time 5 & 0.92 & 0.61 & 1.77 & 2.36 & 1.37 & 0.90 \\
\hline \multicolumn{7}{|l|}{ ODD } \\
\hline Time 1 & 0.72 & 0.49 & 1.39 & 1.62 & 1.29 & 1.24 \\
\hline Time 2 & 0.80 & 0.58 & 1.54 & 1.93 & 1.32 & 1.28 \\
\hline Time 3 & 0.84 & 0.59 & 1.56 & 1.99 & 1.34 & 1.15 \\
\hline Time 4 & 0.77 & 0.58 & 1.39 & 1.94 & 1.53 & 1.68 \\
\hline Time 5 & 0.83 & 0.59 & 1.39 & 2.07 & 1.73 & 2.25 \\
\hline
\end{tabular}


Table 2

Fit Statistics for Trajectories of ADHD and ODD

\begin{tabular}{llll}
\hline & AIC & BIC & $n$ per class \\
\hline Hyperactivity & & & \\
3-class linear & 3740.94 & 3791.47 & $43,129,1$ \\
4-class linear & 3728.86 & 3790.22 & $9,36,91,137$ \\
3-class quadratic & 3715.85 & 3777.21 & $178,72,23$ \\
4-class quadratic & 3707.43 & 3783.23 & $76,24,130,43$ \\
Inattention & & & \\
3-class linear & 3587.89 & 3638.42 & $76,177,20$ \\
4-class linear & 3563.48 & 3624.84 & $39,19,179,36$ \\
3-class quadratic & 3561.94 & 3623.31 & $207,51,15$ \\
4-class quadratic & 3513.34 & 3589.14 & $11,32,191,39$ \\
ODD & & & \\
3-class linear & 3374.24 & 3424.77 & $18,15,240$ \\
4-class linear & 3328.01 & 3389.37 & $13,199,37,24$ \\
3-class quadratic & 3345.32 & 3406.68 & $8,24,241$ \\
4-class quadratic & 3307.73 & 3383.53 & $207,8,17,41$ \\
\hline
\end{tabular}


Table 3

Summary of Final Trajectory Models

$\begin{array}{llll}\text { T1 intercept } & \text { T1 slope } & \text { Quadratic } & N(\%) \\ M(\mathrm{SE}) & M(S E) & \text { term } & \\ & & M(S E) & \end{array}$

\begin{tabular}{|c|c|c|c|c|}
\hline \multicolumn{5}{|l|}{ Hyperactivity } \\
\hline Class $1 /$ High & $5.10(.30)^{* * *}$ & $.97(.24)^{* * *}$ & $-.27(.06)^{* * *}$ & $76(28 \%)$ \\
\hline Class 2/Moderate increasing & $3.53(.56)^{* * *}$ & $-.20(.38)$ & $.26(.08)^{* *}$ & $24(9 \%)$ \\
\hline Class 3/Low & $1.57(.21)^{* * *}$ & $-0.28(.21)$ & $.05(.04)$ & $\begin{array}{l}130 \\
(48 \%)\end{array}$ \\
\hline Class 4/Declining & $4.69(.35)^{* * *}$ & $-2.18(.27)^{* * *}$ & $.33(.06)^{* * *}$ & $43(16 \%)$ \\
\hline \multicolumn{5}{|l|}{ Inattention } \\
\hline Class $1 /$ Moderate fast increasing & $3.59(1.05)^{* *}$ & $2.92(.83)^{* * *}$ & $-.44(.16)^{* *}$ & $11(4 \%)$ \\
\hline Class 2/High-moderate & $5.07(.77)^{* * *}$ & $-.32(.44)$ & $-.06(.10)$ & $32(12 \%)$ \\
\hline Class 3/Low-declining & $1.30(.14)^{* * *}$ & $-.51(.14)^{* * *}$ & $.08(.03)^{* * *}$ & $\begin{array}{l}191 \\
(70 \%)\end{array}$ \\
\hline $\begin{array}{l}\text { Class 4/Moderate slow } \\
\text { increasing } \\
\text { ODD }\end{array}$ & $3.15(.40)^{* * * *}$ & $-1.17(.51)^{* *}$ & $.42(.12)^{* * *}$ & $39(14 \%)$ \\
\hline Class 1/Low stable & $1.09(.13)^{* * *}$ & $-.08(.16)$ & $-.02(.04)$ & $\begin{array}{l}207 \\
(76 \%)\end{array}$ \\
\hline Class 2/Low increasing & $1.28(.38)^{* *}$ & $-1.17(.47)^{*}$ & $.64(.11)^{* * *}$ & $8(3 \%)$ \\
\hline Class 3/High increasing & $3.99(.78)^{* * *}$ & $1.62(.76)^{*}$ & $.25(.16)$ & $17(6 \%)$ \\
\hline Class 4/Moderate & $1.80(.38)^{* * *}$ & $.79(.60)$ & $-.13(.14)$ & $41(15 \%)$ \\
\hline
\end{tabular}

Note. Rounded to the nearest whole percentage so total numbers might exceed $100 \%$. $* * * p<.001 ; * * p<.01 ; * p<.05$ 
Table 4

LCA with Temperament Traits as Covariates

\begin{tabular}{|c|c|c|c|c|c|c|c|c|c|}
\hline & \multicolumn{3}{|c|}{ Surgency } & \multicolumn{3}{|c|}{ Effortful Control $^{\mathrm{a}}$} & \multicolumn{3}{|c|}{ Negative Affect } \\
\hline & $b(\mathrm{SE})$ & $p$ & OR & $b(\mathrm{SE})$ & $p$ & OR & $b(\mathrm{SE})$ & $p$ & OR \\
\hline \multicolumn{10}{|l|}{ Domain } \\
\hline \multicolumn{10}{|l|}{ Hyperactivity } \\
\hline High vs. low & $3.04(.82)$ & $<.001$ & 20.91 & $2.14(.49)$ & $<.001$ & 8.50 & $1.12(.41)$ & .006 & 3.06 \\
\hline High vs. declining & $-0.83(.65)$ & .200 & 0.44 & $0.79(.44)$ & .067 & 2.20 & $0.41(.58)$ & .488 & 1.51 \\
\hline High vs. mod-increasing & $2.31(.86)$ & .007 & 10.07 & $1.35(.50)$ & .007 & 3.86 & $0.37(.49)$ & .446 & 1.45 \\
\hline Declining vs. low & $2.21(.65)$ & .001 & 9.12 & $1.34(.59)$ & .022 & 3.82 & $0.71(.65)$ & .272 & 2.03 \\
\hline Mod-increasing vs. low & $0.72(.42)$ & .084 & 2.05 & $0.79(.49)$ & .107 & 2.20 & $0.74(.48)$ & .123 & 2.10 \\
\hline $\begin{array}{l}\text { Declining vs. mod- } \\
\text { increasing }\end{array}$ & $1.49(.67)$ & .026 & 4.44 & $0.55(.56)$ & .323 & 1.73 & $-0.03(.62)$ & .960 & 0.97 \\
\hline \multicolumn{10}{|l|}{ Inattention } \\
\hline High-mod vs. low & $1.99(.61)$ & .001 & 7.32 & $1.66(.35)$ & $<.001$ & 5.26 & $1.14(.46)$ & .014 & 3.13 \\
\hline $\begin{array}{l}\text { High-mod vs. mod-fast- } \\
\text { increasing }\end{array}$ & $0.63(.77)$ & .409 & 1.88 & $0.46(.79)$ & .560 & 1.58 & $0.23(.64)$ & .720 & 1.26 \\
\hline $\begin{array}{l}\text { High-mod vs. mod-slow- } \\
\text { increasing }\end{array}$ & $1.43(.76)$ & .061 & 4.18 & $0.60(.38)$ & .120 & 1.82 & $0.03(.54)$ & .964 & 1.02 \\
\hline $\begin{array}{l}\text { Mod-slow- increasing vs. } \\
\text { low }\end{array}$ & $0.56(.47)$ & .227 & 1.75 & $1.06(.35)$ & .002 & 2.89 & $1.11(.45)$ & .014 & 3.03 \\
\hline Mod-fast-increasing vs. low & $1.36(.51)$ & .007 & 3.90 & $1.20(.69)$ & .085 & 3.32 & $0.91(.55)$ & .099 & 2.48 \\
\hline $\begin{array}{l}\text { Mod-slow-increasing vs. } \\
\text { mod-fast increasing }\end{array}$ & $-0.79(.63)$ & .205 & 0.45 & $-.14(.73)$ & .853 & 0.87 & $0.21(.59)$ & .729 & 1.23 \\
\hline \multicolumn{10}{|l|}{ ODD } \\
\hline High-increasing vs. low & $1.74(.53)$ & .001 & 5.70 & $1.52(.44)$ & .001 & 4.57 & $1.98(.58)$ & .001 & 7.24 \\
\hline
\end{tabular}




\begin{tabular}{|c|c|c|c|c|c|c|c|c|c|}
\hline $\begin{array}{l}\text { High-increasing vs. low- } \\
\text { increasing }\end{array}$ & $1.10(.67)$ & .101 & 3.00 & $0.95(.66)$ & .150 & 2.59 & $2.04(1.15)$ & .077 & 7.69 \\
\hline High-increasing vs. mod & $0.77(.71)$ & .276 & 2.16 & $-.65(.47)$ & .168 & 0.52 & $0.94(.67)$ & .161 & 2.56 \\
\hline Mod vs. low & $0.97(.47)$ & .041 & 2.64 & $0.87(.34)$ & .010 & 2.39 & $1.04(.47)$ & .027 & 2.83 \\
\hline Low-increasing vs. low & $0.64(.48)$ & .185 & 1.90 & $0.57(.57)$ & .318 & 1.77 & $-0.06(1.03)$ & .950 & 0.94 \\
\hline Mod vs. low-increasing & $0.33(.64)$ & .602 & 1.39 & $-.30(.61)$ & .623 & 0.74 & $1.10(1.10)$ & .318 & 3.00 \\
\hline
\end{tabular}

Note. Significant results are bolded. OR = Odds Ratio; Mod = moderate.

${ }^{\text {a}}$ Reverse direction of class vs. reference class reported (e.g., moderate vs. low is reported with moderate as the comparison group) 
Table 5

Dual Trajectory Results for ADHD and ODD Trajectory Classes

\begin{tabular}{|c|c|c|c|c|}
\hline \multirow[t]{2}{*}{ ADHD Class } & \multicolumn{4}{|c|}{ ODD Class } \\
\hline & Low $^{\mathrm{a}}$ & $\begin{array}{l}\text { Low- } \\
\text { increasing }\end{array}$ & Moderate & $\begin{array}{l}\text { High- } \\
\text { increasing }\end{array}$ \\
\hline \multicolumn{5}{|c|}{ Probability of ODD Conditional on Hyperactivity/impulsivity } \\
\hline Low & .867 & .000 & .113 & .020 \\
\hline Declining & .685 & .041 & .274 & .000 \\
\hline Moderate-increasing & .365 & .335 & .300 & .000 \\
\hline High & .111 & .061 & .559 & .269 \\
\hline \multicolumn{5}{|c|}{ Probability of Hyperactivity/impulsivity Conditional on ODD } \\
\hline Low & .672 & .000 & .191 & .122 \\
\hline Declining & .218 & .134 & .190 & .000 \\
\hline Moderate-increasing & .064 & .604 & .114 & .000 \\
\hline High & .046 & .262 & .505 & .878 \\
\hline \multicolumn{5}{|c|}{ Probability of ODD Conditional on Inattention } \\
\hline Low-declining & .834 & .032 & .127 & .007 \\
\hline Moderate-slow-increasing & .270 & .147 & .482 & .101 \\
\hline Moderate-fast-increasing & .170 & .133 & .193 & .504 \\
\hline High & .169 & .000 & .620 & .211 \\
\hline \multicolumn{5}{|c|}{ Probability of Inattention Conditional on ODD } \\
\hline Low-declining & .878 & .393 & .332 & .063 \\
\hline Moderate-slow-increasing & .013 & .118 & .036 & .335 \\
\hline Moderate-fast-increasing & .077 & .489 & .338 & .250 \\
\hline High & .032 & .000 & .293 & .352 \\
\hline
\end{tabular}

${ }^{\mathrm{a}}$ For some of these analyses, the low class was declining over time. 
Table 6

Dual Trajectory Results for Hyperactivity/impulsivity and Inattention Classes

\begin{tabular}{|c|c|c|c|c|}
\hline & \multicolumn{4}{|c|}{ Hyperactivity/impulsivity Class } \\
\hline Inattention Class $^{\mathrm{a}}$ & Low & Moderate-stable & Declining & High \\
\hline \multicolumn{5}{|c|}{ Probability of Inattention Conditional on Hyperactivity/impulsivity } \\
\hline Low & .965 & .466 & .000 & .055 \\
\hline Moderate & .035 & .332 & .000 & .366 \\
\hline Moderate-slow-increasing & .000 & .202 & .739 & .230 \\
\hline High & .000 & .000 & .261 & .349 \\
\hline \multicolumn{5}{|c|}{ Probability of Hyperactivity/impulsivity Conditional on Inattention } \\
\hline Low & .755 & .235 & .000 & .011 \\
\hline Moderate & .113 & 603 & .000 & .284 \\
\hline Moderate-slow-increasing & .000 & .316 & .402 & .282 \\
\hline High & .000 & .000 & .080 & .920 \\
\hline
\end{tabular}

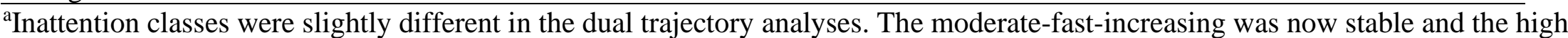
group was increasing at Time 1 . 


\section{BIBLIOGRAPHY}

American Psychiatric Association (2013). Diagnostic and statistical manual of mental disorders, $5^{\text {th }}$ edition (DSM-5). Washington, D.C.: American Psychiatric Association.

Anderson, T. N., \& Kida, T. E. (1982). The cross-lagged research approach: description and illustration. Journal of Accounting Research, 20(2), 403-414. https://doi.org/10.2307/ 2490748

Asparouhov, T., \& Muthén, B. (2014a). Auxiliary variables in mixture modeling: Using the BCH method in Mplus to estimate a distal outcome model and an arbitrary secondary model. Mplus Web Notes, 21(2), 1-22. Retrieved from: https://www.statmodel.com/ download/asparouhov_muthen_2014.pdf https://doi.org/10.1080/10705511.2014.915181

Asparouhov, T., \& Muthén, B. (2014b). Auxiliary variables in mixture modeling: Three-step Approaches Using Mplus. Mplus Web Notes, 21(3), 329-341. https://doi.org/10.1080/ 10705511.2014.915181

Atherton, O. E., Lawson, K. M., Ferrer, E., \& Robins, R. W. (2019, March 28). The Role of Effortful Control in the Development of ADHD, ODD, and CD Symptoms. Journal of Personality and Social Psychology. Advance online publication. https://doi.org/10.1037/ pspp0000243

Azeredo, A., Moreira, D., \& Barbosa, F. (2018). ADHD, CD, and ODD: Systematic review of genetic and environmental risk factors. Research in developmental disabilities, 82, 10-19. https://doi.org/10.1016/j.ridd.2017.12.010

Baillargeon, R. H., Zoccolillo, M., Keenan, K., Côté, S., Pérusse, D., Wu, H. X., ... \& Tremblay, R. E. (2007). Gender differences in physical aggression: A prospective population-based survey of children before and after 2 years of age. Developmental psychology, 43(1), 13. https://doi.org/10.1037/0012-1649.43.1.13

Barkley, R.A. (1998). Attention-deficit hyperactivity disorder: A handbook for diagnosis and treatment. New York: Guilford

Berger, I., \& Nevo, Y. (2011). Early developmental cues for diagnosis of attention deficit/hyperactivity disorder in young children. Developmental Disabilities Research Reviews, 17, 170-179. https://doi.org/10.1002/ddrr.1111

Besemer, S., Loeber, R., Hinshaw, S. P., \& Pardini, D. A. (2016). Bidirectional associations between externalizing behavior problems and maladaptive parenting within parent-son dyads across childhood. Journal of Abnormal Child Psychology, 44, 1387-1398. https://doi.org/10.1007/s10802-015-0124-6 
Bornovalova, M. A., Hicks, B. M., Iacono, W. G., \& McGue, M. (2010). Familial transmission and heritability of childhood disruptive disorders. The American Journal of Psychiatry, 167, 1066-1074. https://doi.org/10.1176/appi.ajp.2010.09091272

Brown, H. R., \& Harvey, E. A. (2019). Psychometric properties of ADHD symptoms in toddlers. Journal of Clinical Child \& Adolescent Psychology, 48(3), 423-439. https://doi.org/10.1080/15374416.2018.1485105

Buhrmester, M., Kwang, T., \& Gosling, S. D. (2011). Amazon's Mechanical Turk: A new source of inexpensive, yet high-quality, data? Perspectives on Psychological Science, 6(1), 3-5. doi: $10.1177 / 1745691610393980$

Burke, J. D., Boylan, K., Rowe, R., Duku, E., Stepp, S. D., Hipwell, A. E., \& Waldman, I. D. (2014). Identifying the irritability dimension of ODD: Application of a modified bifactor model across five large community samples of children. Journal of Abnormal Psychology, 123(4), 841. https://doi.org/10.1037/a0037898

Burke, J. D., Hipwell, A. E., \& Loeber, R. (2010). Dimensions of oppositional defiant disorder as predictors of depression and conduct disorder in preadolescent girls. Journal of the American Academy of Child \& Adolescent Psychiatry, 49(5), 484-492. https://doi.org/ 10.1016/j.jaac.2010.01.016

Burke, J. D., Loeber, R., Lahey, B. B., \& Rathouz, P. J. (2005). Developmental transitions among affective and behavioral disorders in adolescent boys. Journal of Child Psychology and Psychiatry, 46(11), 1200-1210. https://doi.org/10.1111/j.14697610.2005.00422.x

Burns, G. L., \& Walsh, J. A. (2002). The influence of ADHD-hyperactivity/impulsivity symptoms on the development of oppositional defiant disorder symptoms in a 2-year longitudinal study. Journal of abnormal child psychology, 30(3), 245-256. https://doi.org/ 10.1023/A:1015102812958

Busemeyer, J. R., \& Diederich, A. (2014). Estimation and testing of computational psychological models. In Neuroeconomics (pp. 49-61). Academic Press.

Campbell, S.B. (1990). Behavior problems in preschool children: Clinical and developmental issues. New York: Guilford Press.

Campbell, S. B., \& Ewing, L. J. (1990). Follow-up of hard-to-manage preschoolers: adjustment at age 9 and predictors of continuing symptoms. Journal of Child Psychology and Psychiatry, 31(6), 871-889. https://doi.org/10.1111/j.1469-7610.1990. tb00831.x

Campbell, S. B., Ewing, L. J., Breaux, A. M., \& Szumowski, E. K. (1986). Parent-referred problem three-year-olds: Follow-up at school entry. Journal of Child Psychology and Psychiatry, 27(4), 473-488. https://doi.org/10.1111/j.1469-7610.1986.tb00635.x 
Campbell, S. B., Szumowski, E. K., Ewing, L. J., Gluck, D. S., \& Breaux, A. M. (1982). A multidimensional assessment of parent-identified behavior problem toddlers. Journal of Abnormal Child Psychology, 10(4), 569-592. https://doi.org/10.1007/BF00920755

Cavanagh, M., Quinn, D., Duncan, D., Graham, T., \& Balbuena, L. (2017). Oppositional defiant disorder is better conceptualized as a disorder of emotional regulation. Journal of attention disorders, 21(5), 381-389. https://doi.org/10.1177/1087054713520221

Clark, Shaunna \& Muthén, Bengt. (2009). Relating Latent Class Analysis Results to Variables not Included in the Analysis. Retrieved from: https://www.researchgate.net/publication/ 237346694_Relating_Latent_Class_Analysis_Results_to_Variables_not_Included_in_the _Analysis

Coates J., Taylor J. A., \& Sayal, K. (2016). Parenting interventions for ADHD: A systematic literature review and meta-analysis. Journal of Attention Disorders, 19, 831-843. doi:10.1177/1087054714535952

Coles, E. K., Slavec, J., Bernstein, M., \& Baroni, E. (2012). Exploring the gender gap in referrals for children with ADHD and other disruptive behavior disorders. Journal of Attention Disorders, 16(2), 101-108. https://doi.org/10.1177/ 1087054710381481

Côté, S. M., Vaillancourt, T., Leblanc, J. C., Nagin, D. S., \& Tremblay, R. E. (2006). The development of physical aggression from toddlerhood to pre-adolescence: A nationwide longitudinal study of Canadian children. Journal of Abnormal Child Psychology, 34(1), 71-85. doi:10.1007/s10802-005-9001-z

de la Osa, N., Penelo, E., Navarro, J. B., Trepat, E., \& Ezpeleta, L. (2019). Prevalence, comorbidity, functioning and long-term effects of subthreshold oppositional defiant disorder in a community sample of preschoolers. European child \& adolescent psychiatry, 1-9. https://doi.org/10.1007/s00787-019-01300-0

Demmer, D. H., Hooley, M., Sheen, J., McGillivray, J. A., \& Lum, J. A. (2017). Sex differences in the prevalence of oppositional defiant disorder during middle childhood: a metaanalysis. Journal of abnormal child psychology, 45(2), 313-325. https://doi.org/ /10.1007/s10802-016-0170-8

Dix, T., Stewart, A. D., Gershoff, E. T., \& Day, W. H. (2007). Autonomy and children's reactions to being controlled: Evidence that both compliance and defiance may be positive markers in early development. Child Development, 78(4), 1204-1221. https://doi.org/10.1111/j.1467-8624.2007.01061.x

DuPaul, G. J., Reid, R., Anastopoulos, A. D., Lambert, M. C., Watkins, M.W., Power, T. J. (2016). Parent and teacher ratings of attention-deficit/hyperactivity disorder symptoms: Factor structure and normative data. Psychological Assessment, 28(2), 214-225. https://doi.org/10.1037/pas0000166 
Dziak, J. J., Lanza, S. T., \& Tan, X. (2014). Effect size, statistical power, and sample size requirements for the bootstrap likelihood ratio test in latent class analysis. Structural equation modeling: a multidisciplinary journal, 21(4), 534-552. https://doi.org/ 10.1080/10705511. 2014.919819

Elia, J., Ambrosini, P., \& Berrettini, W. (2008). ADHD characteristics: I. Concurrent comorbidity patterns in children \& adolescents. Child and adolescent psychiatry and mental health, 2(1), 15. https://doi.org/10.1186/1753-2000-2-15

Erikson, E.H. (1963). Childhood and Society. New York, NY: Norton

Faraone, S. V., Asherson, P., Banaschewski, T., Biederman, J., Buitelaar, J. K., Ramos-Quiroga, J. A., \& Franke, B. (2015). Attention-deficit/hyperactivity disorder. Nature Reviews: Disease Primers, 1, 15020. https://doi.org/10.1038/nrdp.2015.20

Fontaine, N., Carbonneau, R., Barker, E. D., Vitaro, F., Hébert, M., Côté, S. M., ... \& Tremblay, R. E. (2008). Girls' hyperactivity and physical aggression during childhood and adjustment problems in early adulthood: a 15-year longitudinal study. Archives of General Psychiatry, 65(3), 320-328. https://doi.org/10.1001/archgenpsychiatry.2007.41

Fox, R. A., Keller, K. M., Grede, P. L., \& Bartosz, A. M. (2007). A mental health clinic for toddlers with developmental delays and behavior problems. Research in Developmental Disabilities, 28(2), 119-129. https://doi.org/10.1016/j.ridd.2006.02.001

Friedman-Weieneth, J. L., Doctoroff, G. L., Harvey, E. A., \& Goldstein, L. H. (2009). The disruptive behavior rating scale - parent version (DBRS-PV) factor analytic structure and validity among young preschool children. Journal of Attention Disorders, 13(1), 42-55. https://doi.org/10.1177/1087054708322991

Galán, C. A., Wang, F. L., Shaw, D. S., \& Forbes, E. E. (2019). Early childhood trajectories of conduct problems and hyperactivity/attention problems: predicting adolescent and adult antisocial behavior and internalizing problems. Journal of Clinical Child \& Adolescent Psychology, 1-15. https://doi.org/10.1080/15374416.2018.1534206

Galéra, C., Coté, S. M., Bouvard, M. P., Pingault, J-B., Melchio, M., Michel, G., ... Tremblay, R. E. (2011). Early risk factors for hyperactivity-impulsivity and inattention trajectories from age 17 months to 8 years. Archives of General Psychiatry, 68(12), 1267-1275. https://doi.org/10.1001/archgenpsychiatry.2011.138

Graziano, P. A., \& Garcia, A. (2016). Attention-deficit hyperactivity disorder and children's emotion dysregulation: A meta-analysis. Clinical Psychology Review, 46, 106-123. https://10.1016/j.cpr.2016.04.011 
Greene, R. W., Biederman, J., Zerwas, S., Monuteaux, M. C., Goring, J. C., \& Faraone, S. V. (2002). Psychiatric comorbidity, family dysfunction, and social impairment in referred youth with oppositional defiant disorder. American Journal of Psychiatry, 159(7), 12141224. https://doi.org/10.1176/appi.ajp.159.7.1214

Gupte-singh, K., Singh, R. R., \& Lawson, K. A. (2017). Economic burden of attention-deficit / hyperactivity disorder among pediatric patients in the United States. Value in Health, 20(4), 602-609. https://doi.org/10.1016/j.jval.2017.01.007

Halvorsen, M., Mathiassen, B., Myrbakk, E., Brøndbo, P. H., Sætrum, A., Steinsvik, O. O., \& Martinussen, M. (2019). Neurodevelopmental correlates of behavioural and emotional problems in a neuropaediatric sample. Research in developmental disabilities, 85, 217 228. Https://doi.org/10.1016/j.ridd.2018.11.005

Hamaker, E. L. (2018). How to run the RI-CLPM with MPlus. Retrieved from: http://www.statmodel.com/download/RI-CLPM\%20Hamaker\%20input.pdf

Hamaker, E. L., Kuiper, R. M., \& Grasman, R. P. P. P. (2015). A critique of the cross-lagged panel model. Psychological Methods, 20, 102-116. https://doi.org/10.1037/a0038889

Harvey, E. A., Breaux, R. P., \& Lugo-Candelas, C. I. (2016). Early development of comorbidity between symptoms of attention-deficit/hyperactivity disorder (ADHD) and oppositional defiant disorder (ODD). Journal of abnormal psychology, 125(2), 154. https://doi.org/ $10.1037 /$ abn0000090

Hommersen, P., Murray, C., Ohan, J. L., \& Johnston, C. (2006). Oppositional defiant disorder rating scale: preliminary evidence of reliability and validity. Journal of Emotional and Behavioral Disorders, 14(2), 118-125. https://doi.org/10.1177/10634266060140020201

Hooper, D., Coughlan, J. and Mullen, M. R. (2008). Structural equation modelling: Guidelines for determining model fit. Electronic Journal of Business Research Methods, 6(1), 5360. Retrieved from: http://arrow.dit.ie/libart/4/

Hu, L., \& Bentler, P.M. (1999). Cutoff criteria for fit indexes in covariance structure analysis: Conventional criteria versus new alternatives. Structural Equation Modeling: A Multidisciplinary Journal, 6(1), 1-55. https://doi.org/10.1080/ 10705519909540118

Huijbregts, S. C. J., Séguin, J. R., Zoccolillo, M., Boivin, M., \& Tremblay, R. E. (2007). Associations of maternal prenatal smoking with early childhood physical aggression, hyperactivity-impulsivity, and their co-occurrence. Journal of Abnormal Child Psychology, 35(2), 203-215. https://doi.org/10.1007/s10802-006-9073-4

Hutchings, J., Griffith, N., Bywater, T., \& Williams, M. E. (2017). Evaluating the Incredible Years Toddler Parenting Programme with parents of toddlers in disadvantaged (Flying Start) areas of Wales. Child: Care, Health and Development, 43, 104-113. doi:10.1111/cch.12415 
Jester, J. M., Nigg, J. T., Adams, K., Fitzgerald, H. E., Puttler, L. I., Wong, M. M., \& Zucker, R. A. (2005). Inattention/hyperactivity and aggression from early childhood to adolescence: Heterogeneity of trajectories and differential influence of family environment characteristics. Development and psychopathology, 17(1), 99-125. https://doi.org/10.1017/ S0954579405050066

Jester, J. M., Nigg, J. T., Buu, A., Puttler, L. I., Glass, J. M., Heitzeg, M. M., ... \& Zucker, R. A. (2008). Trajectories of childhood aggression and inattention/hyperactivity: Differential effects on substance abuse in adolescence. Journal of the American Academy of Child \& Adolescent Psychiatry, 47(10), 1158-1165. https://doi.org/10.1097/CHI. 0b013e3181825a4e

Johnston, C., \& Mash, E. J. (2001). Families of children with attention-deficit/hyperactivity disorder: Review and recommendations for future research. Clinical Child and Family Psychology Review, 4, 183-207. https://doi.org/10.1023/A:1017592030434

Kaiser, N. M., McBurnett, K., \& Pfiffner, L. J. (2011). Child ADHD severity and positive and negative parenting as predictors of child social functioning: Evaluation of three theoretical models. Journal of Attention Disorders, 15(3), 193-203. https://doi.org/ $10.1177 / 1087054709356171$

Keenan, K., \& Wakschlag, L. S. (2000). More than the terrible twos: The nature and severity of behavior problems in clinic-referred preschool children. Journal of Abnormal Child Psychology, 28(1), 33-46. https://doi.org/10.1023/A:1005118000977

Kerekes, N., Lundström, S., Chang, Z., Tajnia, A., Jern, P., Lichtenstein, P., ... \& Anckarsäter, H. (2014). Oppositional defiant-and conduct disorder-like problems: neurodevelopmental predictors and genetic background in boys and girls, in a nationwide twin study. PeerJ, 2, e359. https://doi.org/10.7717/peerj.359

Kochanska, G., Coy, K. C., \& Murray, K. T. (2001). The development of self-regulation in the first four years of life. Child Development, 72(4), 1091-1111. https://doi.org/10.1111/1467-8624.00336

Kochanska, G., \& Kim, S. (2013). Difficult temperament moderates links between maternal responsiveness and children's compliance and behavior problems in low-income families. Journal of Child Psychology and Psychiatry, 54(3), 323-332. https://doi.org/10.1111/ jcpp.12002

Kochanska, G., \& Knaack, A. (2003). Effortful control as a personality characteristic of young children: Antecedents, correlates, and consequences. Journal of Personality, 71(6), 10871112. https://doi.org/10.1111/1467-6494.7106008 
Lahey, B. B., Pelham, W. E., Loney, J., Kipp, H., Ehrhardt, A., Lee, S. S., ... Massetti, G. (2004). Three-year predictive validity of DSM-IV attention deficit hyperactivity disorder in children diagnosed at 4-6 years of age. The American Journal of Psychiatry, 161(11), 2014-2020. https://doi.org/10.1176/appi.ajp.161.11.2014

Lavigne, J. V, Arend, R., Rosenbaum, D., Binns, H. J., Christoffel, K. K., \& Gibbons, R. D. (1998). Psychiatric disorders with onset in the preschool years: Stability of diagnoses. Journal of the American Academy of Child and Adolescent Psychiatry, 37(12), 12461254. https://10.1097/00004583-199812000-00007

Lavigne, J. V., Gibbons, R. D., Christoffel, K. K., Arend, R., Rosenbaum, D., Binns, H., ... Isaacs, C. (1996). Prevalence rates and correlates of psychiatric disorders among preschool children. Journal of the American Academy of Child \& Adolescent Psychiatry, 35(2), 204-214. https://doi.org/10.1097/00004583-199602000-00014

Lavigne, J. V., LeBailly, S. A., Hopkins, J., Gouze, K. R., \& Binns, H. J. (2009). The prevalence of ADHD, ODD, depression, and anxiety in a community sample of 4-year-olds. Journal of Clinical Child \& Adolescent Psychology, 38(3), 315-328. https://doi.org/10.1080/ 15374410902851382

Leaper, C., \& Friedman, C. K. (2007). The Socialization of Gender. In J. E. Grusec \& P. D. Hastings (Eds.), Handbook of socialization: Theory and research (p. 561-587). Guilford Press.

Leblanc, N., Boivin, M., Dionne, G., Brendgen, M., Vitaro, F., Tremblay, R. E., \& Pérusse, D. (2008). The development of hyperactive-impulsive behaviors during the preschool years: The predictive validity of parental assessments. Journal of Abnormal Child Psychology, 36(7), 977-987. https://doi.org/10.1007/s10802-008-9227-7

Li, M., \& Harring, J. R. (2017). Investigating approaches to estimating covariate effects in growth mixture modeling: a simulation study. Educational and psychological measurement, 77(5), 766-791. https://doi.org/10.1177/0013164416653789

Martel, M. M. (2009). Research review: A new perspective on attention-deficit/hyperactivity disorder: Emotion dysregulation and trait models. Journal of Child Psychology and Psychiatry, 50(9), 1042-1051. https://doi.org/10.1111/j.1469-7610.2009.02105.x

Martel, M. M., Nigg, J. T., \& Von Eye, A. (2009). How do trait dimensions map onto ADHD symptom domains?. Journal of Abnormal Child Psychology, 37(3), 337. https://doi.org/ $10.1007 / \mathrm{s} 10802-008-9255-3$

McGoey, K. E., DuPaul, G. J., Haley, E., \& Shelton, T. L. (2007). Parent and teacher ratings of attention-deficit/hyperactivity disorder in preschool: The ADHD Rating Scale-IV Preschool Version. Journal of Psychopathology and Behavioral Assessment, 29(4), 269276. https://doi.org/10.1007/s10862-007-9048-y 
Mesman, J., Stoel, R., Bakermans-Kranenburg, M. J., van IJzendoorn, M. H., Juffer, F., Koot, H. M., \& Alink, L. R. (2009). Predicting growth curves of early childhood externalizing problems: Differential susceptibility of children with difficult temperament. Journal of Abnormal Child Psychology, 37(5), 625. https://doi.org/10.1007/s10802-009-9298-0

Miner, J. L., \& Clarke-Stewart, K. A. (2008). Trajectories of externalizing behavior from age 2 to age 9: relations with gender, temperament, ethnicity, parenting, and rater.

Developmental psychology, 44(3), 771. https://doi.org/10.1037/0012-1649.44.3.771

Murray, K. T., \& Kochanska, G. (2002). Effortful control: Factor structure and relation to externalizing and internalizing behaviors. Journal of Abnormal Child Psychology, 30(5), 503-514. https://doi.org/10.1023/A:1019821031523

Muthén, L. K., \& Muthén, B. O. (1998 - 2017). Mplus: Statistical analysis with latent variables: User's Guide. Eighth edition. Los Angeles, CA: Muthén \& Muthén.

Nagin, D. S. (1999). Analyzing developmental trajectories: A semiparametric, group-based approach. Psychological Methods, 4(2), 139-157. https://doi.org/10.1037/1082989X.4.2.139

Nagin, D. S., \& Odgers, C. L. (2010). Group-based trajectory modeling in clinical research. Annual review of clinical psychology, 6, 109-138. https://doi.org/10.1146/annurev.clinpsy.121208.131413

Nagin, D. S., \& Tremblay, R. E. (2001). Analyzing developmental trajectories of distinct but related behaviors: a group-based method. Psychological methods, 6(1), 18. https://doi.org/10.I037//1082-989X.6.U8

Nelson, T., East, P., Delva, J., Lozoff, B., \& Gahagan, S. (2019). Children's inattention and hyperactivity, mother's parenting, and risk behaviors in adolescence: A 10-year longitudinal study of Chilean children. Journal of Developmental \& Behavioral Pediatrics, 40(4), 249-256. https://doi.org/10.1097/DBP.0000000000000661

Niccols, A. (2009). Immediate and short-term outcomes of the 'COPEing with Toddler Behaviour' parent group. Journal of Child Psychology and Psychiatry, 50(5), 617-626. doi:10.1111/j.1469-7610.2008.02007.x

Nigg, J. T., John, O. P., Blaskey, L. G., Huang-Pollock, C. L., Willcutt, E. G., Hinshaw, S. P., \& Pennington, B. (2002). Big five dimensions and ADHD symptoms: Links between personality traits and clinical symptoms. Journal of Personality and Social psychology, 83(2), 451. https://doi.org/10.1037/0022-3514.83.2.451

Nylund, K. L., Asparouhov, T., \& Muthén, B. O. Deciding on the number of classes in latent class analysis and growth mixture modeling: A Monte Carlo simulation study. Structural equation modeling: A multidisciplinary Journal 14.4 (2007): 535-569. https://doi.org/ 10.1080/10705510701575396 
Owens, E. B., \& Shaw, D. S. (2003). Predicting growth curves of externalizing behavior across the preschool years. Journal of Abnormal Child Psychology, 31(6), 575-90.

https://doi.org/10.1023/A:1026254005632

Palfrey, J. S., Levine, M. D., Walker, D. K., \& Sullivan, M. (1985). The emergence of attention deficits in early childhood: A prospective study. Journal of Developmental and Behavioral Pediatrics, 6(6), 339-348. Retrieved from: http://www.ncbi.nlm. nih.gov/pubmed/4077993

Petitclerc, A., Boivin, M., Dionne, G., Zoccolillo, M., \& Tremblay, R. E. (2009). Disregard for rules: The early development and predictors of a specific dimension of disruptive behavior disorders. Journal of Child Psychology and Psychiatry, 50(12), 1477-1484. https://doi.org/10.1111/j.1469-7610.2009.02118.x

Pierce, E. W., Ewing, L. J., \& Campbell, S.B. (1999). Diagnostic status and symptomatic behavior of hard-to-manage preschool children in middle childhood and early adolescence. Journal of Clinical Child Psychology, 28(1), 44-57. Retrieved from http://www.ncbi.nlm.nih.gov/pubmed/10070606

Putnam, S. P., Jacobs, J., Gartstein, M. A., \& Rothbart, M. K. (2010, March). Development and assessment of short and very short forms of the Early Childhood Behavior Questionnaire. Poster presented at the International Conference on Infant Studies, Baltimore, MD.

Rabinovitz, B. B., O'Neill, S., Rajendran, K., \& Halperin, J. M. (2016). Temperament, executive control, and attention-deficit/hyperactivity disorder across early development. Journal of Abnormal Psychology, 125(2), 196. https://doi.org/10.1037/abn0000093

Reale, L., Bartoli, B., Cartabia, M., Zanetti, M., Costantino, M. A., Canevini, M. P., ... \& Lombardy ADHD Group. (2017). Comorbidity prevalence and treatment outcome in children and adolescents with ADHD. European child \& adolescent psychiatry, 26(12), 1443-1457. https://doi.org/10.1007/s00787-017-1005-z

Rhee, S. H., Willcutt, E. G., Hartman, C. A., Pennington, B. F., \& DeFries, J. C. (2008). Test of alternative hypotheses explaining the comorbidity between attention-deficit/hyperactivity disorder and conduct disorder. Journal of abnormal child psychology, 36(1), 29-40. https://doi.org/10.1007/s10802-007-9157-9

Riddle, M. A., Yershova, K., Lazzaretto, D., Paykina, N., Yenokyan, G., Greenhill, L., ... Posner, K. (2013). The Preschool Attention-deficit/hyperactivity disorder Treatment Study (PATS) 6-year follow-up. Journal of the American Academy of Child and Adolescent Psychiatry, 52(3), 264-278. https://doi.org/10.1016/j.jaac.2012.12.007

Romano, E., Tremblay, R. E., Farhat, A., \& Côté, S. (2006). Development and prediction of hyperactive symptoms from 2 to 7 years in a population-based sample. Pediatrics, 117(6), 2101-10. Retrieved from http://www.ncbi.nlm.nih.gov/pubmed/16740853 
Rothbart, M. K., Ahadi, S. A., \& Evans, D. E. (2000). Temperament and personality: origins and outcomes. Journal of personality and social psychology, 78(1), 122. https://doi.org/ 10.1037//0022-3514.78.1.122

Rothbart, M. K., Sheese, B. E., Rueda, M. R., \& Posner, M. I. (2011). Developing mechanisms of self-regulation in early life. Emotion Review, 3(2), 207-213. https://doi.org/10.1177/ 1754073910387943

Rubin, K. H., Burgess, K. B., Dwyer, K. M., \& Hastings, P. D. (2003). Predicting preschoolers' externalizing behaviors from toddler temperament, conflict, and maternal negativity. Developmental psychology, 39(1), 164. https://doi.org/10.1037/00121649.39.1.164

Salla, J., Michel, G., Pingault, J. B., Lacourse, E., Paquin, S., Galéra, C., ... Côté, S. M. (2016). Childhood trajectories of inattention-hyperactivity and academic achievement at 12 years. European Child \& Adolescent Psychiatry. Advance online publication. https://doi.org/ 10.1007/s00787-016-0843-4

Schleider, J. L., \& Weisz, J. R. (2015). Using Mechanical Turk to study family processes and youth mental health: A test of feasibility. Journal of child and family studies, 24(11), 3235-3246. https://doi.org/10.1007/s10826-015-0126-6

Sciutto, M. J., Nolfi, C. J., \& Bluhm, C. (2004). Effects of child gender and symptom type on referrals for ADHD by elementary school teachers. Journal of Emotional and Behavioral Disorders, 12(4), 247-253. https://doi.org/10.1177/10634266040120040501

Seymour, K. E., Chronis-Tuscano, A., Halldorsdottir, T., Stupica, B., Owens, K., \& Sacks, T. (2012). Emotion regulation mediates the relationship between ADHD and depressive symptoms in youth. Journal of Abnormal Child Psychology, 40(4), 595-606. https://doi:10.1007/ s10802-011-9593-4

Sharma, A., \& Couture, J. (2014). A review of the pathophysiology, etiology, and treatment of attention-deficit hyperactivity disorder (ADHD). Annals of Pharmacotherapy, 48(2), 209-225. https://doi.org/10.1177/1060028013510699

Shaw, D. S., Gilliom, M., Ingoldsby, E. M., \& Nagin, D. S. (2003). Trajectories leading to school-age conduct problems. Developmental Psychology, 39(2), 189-200. https://doi.org/10.1037/0012-1649.39.2.189

Shaw, D. S., Lacourse, E., \& Nagin, D. S. (2005). Developmental trajectories of conduct problems and hyperactivity from ages 2 to 10. Journal of Child Psychology and Psychiatry, 46(9), 931-942. https://doi.org/10.1111/j.1469-7610.2004.00390.x 
Shaw, D. S., Owens, E. B., Giovannelli, J., \& Winslow, E. B. (2001). Infant and toddler pathways leading to early externalizing disorders. Journal of the American Academy of Child and Adolescent Psychiatry, 40(1), 36-43. doi:10.1097/00004583-20010100000014

Smith, C. L., \& Day, K. L. (2018). Parenting, anger, and effortful control as predictors of child externalizing behavior: The role of child sex as a moderator. International journal of behavioral development, 42(2), 248-256. https://doi.org/10.1177/0165025417692898

Stifter, C. A., Putnam, S., \& Jahromi, L. (2008). Exuberant and inhibited toddlers: Stability of temperament and risk for problem behavior. Development and psychopathology, 20(2), 401-421. https://doi.org/10.1017/S0954579408000199

Stringaris, A., \& Goodman, R. (2009). Longitudinal outcome of youth oppositionality: irritable, headstrong, and hurtful behaviors have distinctive predictions. Journal of the American Academy of Child \& Adolescent Psychiatry, 48(4), 404-412. https://doi.org/10.1097/CHI. 0b013e3181984f30

Stringaris, A., Maughan, B., \& Goodman, R. (2010). What's in a disruptive disorder? Temperamental antecedents of oppositional defiant disorder: findings from the Avon longitudinal study. Journal of the American Academy of Child \& Adolescent Psychiatry, 49(5), 474-483. https://doi.org/10.1016/j.jaac.2010.01.021

Tabachnick, B. G., \& Fidell, L. S. (2013). Using Multivariate Statistics, $\sigma^{\text {th }}$ ed. Boston: Allyn and Bacon.

Tackett, J. L. (2006). Evaluating models of the personality-psychopathology relationship in children and adolescents. Clinical Psychology Review, 26, 584-599. https://doi.org/10. 1016/j.cpr.2006.04.003

Thoemmes, F., MacKinnon, D. P., \& Reiser, M. R. (2010). Power analysis for complex mediational designs using Monte Carlo methods. Structural Equation Modeling, 17(3), 510-534. https://doi.org/10.1080/10705511.2010.489379

Tiego, J., Bellgrove, M. A., Whittle, S., Pantelis, C., \& Testa, R. (2020). Common mechanisms of executive attention underlie executive function and effortful control in children. Developmental science, 23(3), e12918. https://doi.org/10.1111/desc.12918

Tremblay, R. E., Nagin, D. S., Seguin, J. R., Zoccolillo, M., Zelazo, P. D., Boivin, M., ... \& Japel, C. (2004). Physical aggression during early childhood: Trajectories and predictors. Pediatrics, 114(1), e43-e50. https://doi.org/10.1542/peds.114.1.e43

Tuvblad, C., Zheng, M., Raine, A., \& Baker, L. A. (2009). A common genetic factor explains the covariation among ADHD ODD and CD symptoms in 9-10 year old boys and girls. Journal of abnormal child psychology, 37(2), 153-167. https://doi.org/ 10.1007/s10802-008-9278-9 
Vermunt, J. K. (2010). Latent class modeling with covariates: Two improved three-step approaches. Political analysis, 18(4), 450-469. https://doi.org/10.1093/pan/mpq025

Wakschlag, L. S., Choi, S. W., Carter, A. S., Hullsiek, H., Burns, J., McCarthy, K., ... BriggsGowan, M. J. (2012). Defining the developmental parameters of temper loss in early childhood: Implications for developmental psychopathology. Journal of Child Psychology and Psychiatry and Allied Disciplines, 53(11), 1099-1108. https://doi.org/10.1111/j.1469-7610.2012.02595.x

Wichstrøm, L., Penelo, E., Rensvik Viddal, K., de la Osa, N., \& Ezpeleta, L. (2018). Explaining the relationship between temperament and symptoms of psychiatric disorders from preschool to middle childhood: hybrid fixed and random effects models of Norwegian and Spanish children. Journal of Child Psychology and Psychiatry, 59(3), 285-295. https://doi.org/10.1111/jcpp.12772

Willoughby, M.T., Pek, J., Greenberg, M. T., \& Family Life Project Investigators (2012). Parentreported attention deficit/hyperactivity symptomatology in preschool-aged children: Factor structure, developmental change, and early risk factors. Journal of Abnormal Child Psychology, 40(8), 1301-1312. https://doi.org/10.1007/s10802-012-9641-8 